TRANSACTIONS OF THE

AMERICAN MATHEMATICAL SOCIETY

Volume 359, Number 4, April 2007, Pages 1605-1632

S 0002-9947(06)04110-9

Article electronically published on October 17, 2006

\title{
NUMERICAL GODEAUX SURFACES WITH AN INVOLUTION
}

\author{
ALBERTO CALABRI, CIRO CILIBERTO, AND MARGARIDA MENDES LOPES
}

\begin{abstract}
Minimal algebraic surfaces of general type with the smallest possible invariants have geometric genus zero and $K^{2}=1$ and are usually called numerical Godeaux surfaces. Although they have been studied by several authors, their complete classification is not known.

In this paper we classify numerical Godeaux surfaces with an involution, i.e. an automorphism of order 2 . We prove that they are birationally equivalent either to double covers of Enriques surfaces or to double planes of two different types: the branch curve either has degree 10 and suitable singularities, originally suggested by Campedelli, or is the union of two lines and a curve of degree 12 with certain singularities. The latter type of double planes are degenerations of examples described by $\mathrm{Du}$ Val, and their existence was previously unknown; we show some examples of this new type, also computing their torsion group.
\end{abstract}

\section{INTRODUCTION}

In the one-and-a-half century history of classification of algebraic varieties, surfaces having geometric genus $p_{g}=0$ and irregularity $q=0$ have been studied from the very beginning. They were conjectured to be rational by Max Noether (about 1870) until Enriques, in 1894, suggested the existence of surfaces with $p_{g}=q=0$ and bi-genus $P_{2}=1$ which now bear his name. After that, Castelnuovo, in 1896, proved his celebrated rationality criterion, which says that a surface $X$ is rational if and only if $P_{2}(X)=q(X)=0$. Since then, the classification of surfaces with $p_{g}=0$ has received particular attention by algebraic geometers, as well as others. Indeed, in the course of the years, it has been discovered that these surfaces are interesting not only for classification purposes, but also for their intriguing relations with other fields of mathematics, e.g. Bloch's conjecture, classification of four-folds, etc. (see B1, Fr; for general information cf. Ci2 ).

In 1931-32, Godeaux and Campedelli gave the first two examples of minimal surfaces of general type with $p_{g}=0$. Godeaux considered a quotient of a quintic surface in $\mathbb{P}^{3}$ by a freely acting cyclic group of order 5 of projective transformations. The smooth minimal model of this surface has $K^{2}=1$. Campedelli constructed a double plane, i.e. a double cover of $\mathbb{P}^{2}$, branched along a degree 10 curve with six

Received by the editors January 19, 2005.

2000 Mathematics Subject Classification. Primary 14J29.

Key words and phrases. Godeaux surface, involution, torsion group.

This research has been carried out in the framework of the EAGER project financed by the EC, project n. HPRN-CT-2000-00099. The first two authors are members of G.N.S.A.G.A.I.N.d.A.M., which generously supported this research. The third author is a member of the Center for Mathematical Analysis, Geometry and Dynamical Systems, IST, and was partially supported by FCT (Portugal) through program POCTI/FEDER and Project POCTI/MAT/44068/2002. 
points, not lying on a conic, all of type [3,3], that is, a triple point with another infinitely near triple point. Here the smooth minimal model has $K^{2}=2$.

Campedelli also proposed the construction of a minimal surface of general type with $p_{g}=0$ and $K^{2}=1$ as the minimal model of a double plane branched along a curve of degree 10 with a 4 -tuple point and five points of type $[3,3]$, not lying on a conic. The actual existence of such a curve was proved only 50 years later by Kulikov, Oort and Peters in $\mathrm{OP}$. We will say that a double plane with branch curve having the singularities suggested by Campedelli is of Campedelli type.

Minimal surfaces of general type with $p_{g}=0$ and $K^{2}=1$ are nowadays called numerical Godeaux surfaces. They have been studied classically, but also by several authors in the last 30 years: it would be too long to recall here all the relevant contributions, anyway most of them appear in our list of references.

As its construction shows, the original Godeaux's example has non-trivial torsion, more precisely its torsion group is cyclic of order 5 . It is actually a result of Miyaoka in [Mi] that the torsion group of a numerical Godeaux surface is cyclic of order at most 5 and surfaces $S$ with $\operatorname{Tors}(S)=\mathbb{Z} / 5 \mathbb{Z}$ fill up an irreducible component of the moduli space of the expected dimension $8=\chi\left(\mathcal{T}_{S}\right)$, consisting of quotients of quintics in $\mathbb{P}^{3}$ by a $\mathbb{Z} / 5 \mathbb{Z}$-action, as in the original Godeaux's construction.

It has to be expected that the larger the torsion is, the easier the study and the classification of these surfaces. This viewpoint has been pursued by Miles Reid in Re1, who proved that also surfaces with $\operatorname{Tors}(S)=\mathbb{Z} / 3 \mathbb{Z}$ or $\mathbb{Z} / 4 \mathbb{Z}$ fill up an 8-dimensional irreducible component of the moduli space, giving an explicit description of their canonical rings.

Although a few examples of numerical Godeaux surfaces $S$ with no torsion (cf. CG, DW] ) or even simply connected (see [Bar2]), or with $\operatorname{Tors}(S)=\mathbb{Z} / 2 \mathbb{Z}$ (see Bar1, We1, We2 ) are known, neither a classification result, nor a description of the moduli space are known in these cases. Furthermore, all the known examples with no torsion or $\operatorname{Tors}(S)=\mathbb{Z} / 2 \mathbb{Z}$ turn out to possess an involution, i.e. a birational automorphism of order 2 .

This motivates the study of numerical Godeaux surfaces with an involution. A first investigation of this subject has been done by J. Keum and Y. Lee in KL, under the assumption that the bicanonical system has no fixed components. In this paper, we make no assumption of this sort, and we prove the following.

Classification Theorem. A numerical Godeaux surface $S$ with an involution $\sigma$ is birationally equivalent to one of the following:

(1) a double plane of Campedelli type;

(2) a double plane branched along a reduced curve which is the union of two distinct lines $r_{1}, r_{2}$ and a curve of degree 12 with the following singularities:

- the point $q_{0}=r_{1} \cap r_{2}$ of multiplicity 4 ;

- a point $q_{i} \in r_{i}, i=1,2$, of type $[4,4]$, where the tangent line is $r_{i}$;

- further, three points $q_{3}, q_{4}, q_{5}$ of multiplicity 4 and a point $q_{6}$ of type $[3,3]$, such that there is no conic through $q_{1}, \ldots, q_{6}$;

(3) a double cover of an Enriques surface branched along a curve of arithmetic genus 2 .

In case (3), the torsion group of $S$ is $\operatorname{Tors}(S)=\mathbb{Z} / 4 \mathbb{Z}$, whilst in case (2) $\operatorname{Tors}(S)$ is either $\mathbb{Z} / 2 \mathbb{Z}$ or $\mathbb{Z} / 4 \mathbb{Z}$. 
As we said, examples of surfaces of type (1) are known in the literature. Surfaces of type (3) will be called of Enriques type. Examples of such surfaces have been produced by Keum and Naie (cf. [Ke, $[\mathrm{Na}$ ). Double planes as in case (2) are, to the best of our knowledge, new in the literature. They appear to be degenerations of double planes with $p_{g}=4$ and $K^{2}=8$, introduced by Du Val in 1952 (see $[\mathrm{Du}$ ), when classifying surfaces with non-birational bicanonical map (cf. Ci1 for a modern reference). For this reason, we call numerical Godeaux surfaces as in (2) of $D u$ Val type. We give explicit examples of these surfaces, both with torsion group $\mathbb{Z} / 2 \mathbb{Z}$ and $\mathbb{Z} / 4 \mathbb{Z}$, in section 9 .

In order to prove our classification theorem, we proceed as follows. First of all, in section 3, we prove some relevant properties of the fixed locus of an involution acting on a surface of general type with geometric genus zero. In order to do so, we follow ideas contained in joint work of the third author and Rita Pardini (e.g. [MP1]), namely we combine the topological and holomorphic fixed point formulas with Kawamata-Viehweg's vanishing theorem.

Applying these results to numerical Godeaux surfaces $S$ with an involution, in section 4 we give a rather precise description of the fixed locus of the involution and we prove that the bicanonical system is invariant under the involution. This is actually the key ingredient for the proof of the classification and explains why degenerations of $\mathrm{Du}$ Val double planes, which have non-birational bicanonical map, come into play (cf. also recent results of Borrelli in [Bo on the classification of surfaces with non-birational bicanonical map and low invariants).

Since the pencil $\left|2 K_{S}\right|$ is invariant, we can in fact consider its image on the quotient surface under the involution, which is a pencil $\mathcal{D}$ of curves of arithmetic genus 2. Using this, one sees that the quotient surface is either rational or birational to an Enriques surface. The latter case, i.e. the Enriques type, is worked out in section 5, where in particular we prove that numerical Godeaux surfaces of this type have torsion group of order 4 and are birational to the double cover of an Enriques surface branched along a curve of arithmetic genus 2, with at most irrelevant singularities, which moves in a linear system with no fixed component.

In case the quotient of $S$ by the involution is rational, one studies the pencil $\mathcal{D}$ using adjunction. This leads to two different cases: one in which the adjoint to this system is a base-point-free pencil of rational curves, the other in which the adjoint is a pencil of curves of genus 1 . These two cases are analysed separately in sections 6 and 7 respectively. The former case leads to double planes of Campedelli type: it suffices to suitably use the pencil of rational curves to map the quotient surface to $\mathbb{F}_{1}$, and then to the plane. The latter case leads to double planes of $\mathrm{Du}$ Val type. Here the quotient surface is mapped to a weak Del Pezzo surface $X$, i.e. $-K_{X}$ is big and nef, having $K_{X}^{2}=1$ and four disjoint $(-2)$-curves, whose sum is an even divisor in $X$. Rational surfaces with an even set of $(-2)$-curves have been studied by Dolgachev, Mendes Lopes and Pardini in DMP and, more recently, by us in $\mathrm{CCM}$. Indeed we apply the main result in $[\mathrm{CCM}$ to find a suitable birational morphism of the weak Del Pezzo surface $X$ to $\mathbb{P}^{2}$, which realizes the original numerical Godeaux surface as a Du Val double plane.

Then we give more information about the previously unknown case of numerical Godeaux surfaces of Du Val type. In particular, in section 8 , we examine the interplay between reducibility of the branch curve and torsion. Our main result in this direction is Theorem 8.5 which, under some assumptions on the branch curve, 
gives a useful criterion to decide whether the torsion is $\mathbb{Z} / 4 \mathbb{Z}$ or $\mathbb{Z} / 2 \mathbb{Z}$, based on the existence or not of plane curves of degree 8 with suitable singularities.

We remark that our results concern the birational classification of pairs $(S, \sigma)$, where $S$ is a numerical Godeaux surface and $\sigma$ an involution of $S$. We do not treat here, in general, the interesting problem of determining how many involutions can occur on a given numerical Godeaux surface and of which type, according to our classification theorem. However, our results do give some partial information. For instance, if the ramification curve $R$ on $S$ has an irreducible component of genus 2 , then we are in case (3) (see Proposition 7.10). In any case, using the 2-torsion, we give a criterion, i.e. Corollary 8.9 , based on the irreducibility of a certain plane cubic, which allows us to distinguish between the $\mathrm{Du}$ Val and the Campedelli types.

As we said, in section 9, we prove the existence of numerical Godeaux double planes of $\mathrm{Du}$ Val type and we are able to compute the torsion of these examples. We do this using Maple ${ }^{\mathrm{TM}}[\mathrm{Ma}]^{1}$ in two different ways. One way is to find branch curves, with irreducible degree 12 component, which are invariant under a projective automorphism of order 2 of the plane, which is an idea originally due to Stagnaro (cf. $[\mathrm{St}]$ ). The resulting examples turn out to have torsion group $\mathbb{Z} / 4 \mathbb{Z}$.

Another way is to try and find the degree 12 component of the branch curve suitably reducible in a line and an irreducible component of degree 11 . We find examples of this type with torsion $\mathbb{Z} / 2 \mathbb{Z}$ and with torsion $\mathbb{Z} / 4 \mathbb{Z}$, and we prove that the former example does not have a different involution, which makes it a double plane of Campedelli type, therefore it is certainly new in the literature. Although we do not treat moduli problems here, we prove that both our examples vary in families whose images in the moduli space have dimension 5 , and the general member of each family is a Du Val double plane with an irreducible degree 12 component of the branch curve (cf. Corollaries 9.6 and 9.7).

\section{Notation And CONVEntions}

In this section we fix the notation which will be used in this paper.

Let $S$ be a complex projective surface. We set:

$\kappa(S): \quad$ the Kodaira dimension of $S$;

$\chi(\mathcal{F}): \quad$ the Euler characteristic of a sheaf $\mathcal{F}$ on $S$;

$\operatorname{Pic}(S): \quad$ the Picard group of $S$;

Tors $(S)$ : the subgroup of $\operatorname{Pic}(S)$ composed of torsion elements;

$\operatorname{Tors}_{n}(S)$ : the subgroup of $\operatorname{Pic}(S)$ composed of elements of torsion $n$;

$\rho(S): \quad$ the rank of the Néron-Severi group of $S$;

$K_{S}: \quad$ a canonical divisor of $S$;

$p_{g}(S): \quad$ the geometric genus of $S$, that is, $h^{0}\left(S, \mathcal{O}_{S}\left(K_{S}\right)\right)$;

$q(S): \quad$ the irregularity of $S$, that is, $h^{1}\left(S, \mathcal{O}_{S}\right)$;

$P_{m}(S): \quad$ the $m$-th pluri-genus of $S$, that is, $h^{0}\left(S, \mathcal{O}_{S}\left(m K_{S}\right)\right), m \geq 1$.

If $S$ is clear from the context, sometimes we will write $p_{g}, q, K^{2}$, etc., instead of $p_{g}(S), q(S), K_{S}^{2}$, etc.

Let $X$ be a complex projective variety. We denote by $e(X)$ the topological Euler characteristic of $X$ and by $p_{a}(X)$ the arithmetic genus of $X$. Recall that if $D$ is a curve on a surface $S$, then $p_{a}(D)=D\left(D+K_{S}\right) / 2+1$.

\footnotetext{
${ }^{1}$ Maple and Maple V are registered trademarks of Waterloo Maple Inc.
} 
We denote by $\equiv$ the linear equivalence of divisors on a surface and by $\sim$ the numerical equivalence. We usually omit the sign - of the intersection product of two divisors on a surface.

Recall that a $(-1)$-curve is a smooth irreducible rational curve $C$ with $C^{2}=-1$. More generally, ones says that a smooth irreducible rational curve $C$ with $C^{2}=$ $-n<0$ is a $(-n)$-curve.

We say that a divisor $D$ on a surface is nef and big if $D^{2}>0$ and $D E \geq 0$ for every irreducible curve $E$.

If $x$ is a real number, we denote by $[x]$ its integer part, i.e. the largest integer number less than or equal to $x$.

A singular point of type $[m, m]$ on a curve is a point of multiplicity $m$ with an infinitely near point again of multiplicity $m$.

\section{INVOLUTIONS ON SURFACES}

Let $S$ be a smooth, irreducible, projective surface over the field $\mathbb{C}$ of complex numbers. An involution of $S$ is an automorphism $\sigma$ of $S$ of order 2. Remark that if $S$ is a minimal surface of general type, then any birational automorphism is an isomorphism, therefore any birational automorphism of order 2 is an involution. If $X$ is any variety and $\psi: S \rightarrow X$ is a rational map, one says that $\psi$ is composed with the involution $\sigma$ if $\psi \circ \sigma=\psi$.

Given an involution $\sigma$ on $S$, its fixed locus is the union of a smooth, possibly reducible, curve $R$ and of $k$ isolated points $p_{1}, \ldots, p_{k}$.

Let $\pi: S \rightarrow \Sigma:=S / \sigma$ be the quotient map and set $B:=\pi(R)$. The surface $\Sigma$ is normal and $\pi\left(p_{1}\right), \ldots, \pi\left(p_{k}\right)$ are ordinary double points, which are the only singularities of $\Sigma$. In particular, the singularities of $\Sigma$ are canonical and the adjunction formula gives $K_{S} \equiv \pi^{*} K_{\Sigma}+R$.

Let $\epsilon: V \rightarrow S$ be the blowing-up of $S$ at $p_{1}, \ldots, p_{k}$ and let $E_{i}$ be the exceptional curve over $p_{i}, i=1, \ldots, k$. Then $\sigma$ induces an involution $\tilde{\sigma}$ of $V$ whose fixed locus is the union of $R_{0}:=\epsilon^{*}(R)$ and of $E_{1}, \ldots, E_{k}$. Denote by $\tilde{\pi}: V \rightarrow W:=V / \tilde{\sigma}$ the projection onto the quotient and set $B_{0}:=\tilde{\pi}\left(R_{0}\right), C_{i}:=\tilde{\pi}\left(E_{i}\right), i=1, \ldots, k$. The surface $W$ is smooth and the $C_{i}$ are disjoint $(-2)$-curves. Denote by $\eta: W \rightarrow \Sigma$ the map induced by $\epsilon$. The map $\eta$ is the minimal resolution of the singularities of $\Sigma$, and there is a commutative diagram:

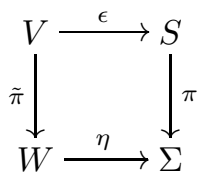

The map $\tilde{\pi}$ is a flat double cover branched on $\tilde{B}=B_{0}+\sum_{i=1}^{k} C_{i}$, hence there exists a divisor $L$ on $W$ such that $2 L \equiv \tilde{B}$ and

$$
\tilde{\pi}_{*} \mathcal{O}_{V}=\mathcal{O}_{W} \oplus \mathcal{O}_{W}(-L)
$$

Also $K_{V} \equiv \tilde{\pi}^{*}\left(K_{W}+L\right)$. With this notation:

Proposition 3.1. Let $S$ be a minimal surface of general type and let $\sigma$ be an involution of $S$. Then:

(i) $2 K_{W}+B_{0}$ is nef and big;

(ii) $\left(2 K_{W}+B_{0}\right)^{2}=2 K_{S}^{2}$;

(iii) $H^{i}\left(W, \mathcal{O}_{W}\left(2 K_{W}+L\right)\right)=0, i=1,2$. 
Proof. By the adjunction formula and commutativity of diagram (10), we have

$$
\tilde{\pi}^{*}\left(2 K_{W}+B_{0}\right) \equiv 2 K_{V}-2 \sum_{i=1}^{k} E_{i} \equiv \epsilon^{*}\left(2 K_{S}\right) .
$$

Then $2 K_{W}+B_{0}$ is nef and big because so is $2 K_{S}$, proving part (i). Statement (ii) also follows by formula (3).

Finally for part (iii), we have the equivalence of $\mathbb{Q}$-divisors:

$$
K_{W}+L \equiv \frac{1}{2}\left(2 K_{W}+B_{0}\right)+\frac{1}{2} \sum_{i=1}^{k} C_{i} .
$$

The divisor $\left(2 K_{W}+B_{0}\right) / 2=\eta^{*}\left(2 K_{\Sigma}+B\right) / 2$ is nef and big, because so is $2 K_{W}+$ $B_{0}$, whereas $\frac{1}{2} \sum_{i=1}^{k} C_{i}$ is effective, with zero integral part, and its support has normal crossings. Thus $h^{i}\left(W, \mathcal{O}_{W}\left(2 K_{W}+L\right)\right)=0$ for $i>0$ by the KawamataViehweg vanishing theorem (see, e.g., Corollary 5.12, c), of [EV]).

For surfaces of general type with $p_{g}=0$ having an involution, one can be more specific. As shown in [KL], Lemma 1.3 or [DMP, the holomorphic and topological fixed point formulas (see p. 566 in $[\mathrm{AS}$ ] and formula (30.9) in $\mathrm{Gr}$ ) yield:

Lemma 3.2. Let $S$ be a surface with $p_{g}=q=0$ and $\sigma$ an involution of $S$. Then the number of isolated fixed points of $\sigma$ is $k=K_{S} R+4$.

Also the following properties, which will be very useful in the sequel, hold.

Proposition 3.3. Let $S$ be a minimal surface of general type with $p_{g}=0$ and let $\sigma$ be an involution of $S$. Then:

(i) $k \geq 4$;

(ii) $K_{W} L+L^{2}=-2$;

(iii) $h^{0}\left(W, \mathcal{O}_{W}\left(2 K_{W}+L\right)\right)=K_{W}^{2}+K_{W} L$;

(iv) $K_{W}^{2}+K_{W} L \geq 0$;

(v) $k=K_{S}^{2}+4-2 h^{0}\left(W, \mathcal{O}_{W}\left(2 K_{W}+L\right)\right)$.

Proof. Since $S$ is minimal of general type, $K_{S}$ is nef, and so statement (i) follows from Lemma 3.2

Since $S$ is of general type with $p_{g}(S)=0$, also $q(S)=0$. Therefore

$$
p_{g}(\Sigma)=p_{g}(W)=0, \quad q(\Sigma)=q(W)=0 .
$$

By standard double cover formulas, we have $\chi\left(\mathcal{O}_{V}\right)=2 \chi\left(\mathcal{O}_{W}\right)+\left(L^{2}+K_{W} L\right) / 2$, thus statement (ii) follows from $p_{g}(W)=q(W)=0$.

By Proposition 3.1(iii), and the Riemann-Roch Theorem, one has

$$
\begin{aligned}
h^{0}\left(W, \mathcal{O}_{W}\left(2 K_{W}+L\right)\right) & =\chi\left(\mathcal{O}_{W}\left(2 K_{W}+L\right)\right)=\left(2 K_{W}+L\right)\left(K_{W}+L\right) / 2+1 \\
& =K_{W}\left(K_{W}+L\right)+L\left(K_{W}+L\right) / 2+1 .
\end{aligned}
$$

Thus (iii) follows by statement (ii), and (iv) is a trivial consequence of (iii).

Finally, as for statement (v), it suffices to remember that $k=K_{S}^{2}-K_{V}^{2}, K_{V}^{2}=$ $2\left(K_{W}+L\right)^{2}$ and use statements (ii), (iii).

Corollary 3.4. Let $S$ be a minimal surface of general type with $p_{g}=0$ and let $\sigma$ be an involution of $S$. Then, with $W$ as above,

(i) $\left|2 K_{W}+B_{0}\right| \neq \emptyset,\left|2 K_{W}+B_{0}+\sum_{i=1}^{k} C_{i}\right|=\left|2 K_{W}+B_{0}\right|+\sum_{i=1}^{k} C_{i}$ and $h^{0}\left(W, \mathcal{O}_{W}\left(2 K_{W}+B_{0}\right)=K_{S}^{2}+1-h^{0}\left(W, \mathcal{O}_{W}\left(2 K_{W}+L\right)\right)\right.$; 
(ii) if $D \in\left|2 K_{W}+B_{0}\right|$, then $D$ is nef and big, 1-connected and thus $h^{0}\left(D, \mathcal{O}_{D}\right)=$ $1, h^{1}\left(D, \mathcal{O}_{D}\right)=p_{a}(D)$

(iii) $p_{a}(D)>0$.

Proof. The Hurwitz formula gives $2 K_{V} \equiv \tilde{\pi}^{*}\left(2 K_{W}+B_{0}+\sum_{i=1}^{k} C_{i}\right)$. By the projection formula (2), one has

$$
H^{0}\left(V, \mathcal{O}_{V}\left(2 K_{V}\right)\right)=H^{0}\left(W, \mathcal{O}_{W}\left(2 K_{W}+L\right)\right) \oplus H^{0}\left(W, \mathcal{O}_{W}\left(2 K_{W}+B_{0}+\sum_{i=1}^{k} C_{i}\right)\right)
$$

By Proposition 3.3(v) and (i), $K_{S}^{2}-2 h^{0}\left(W, \mathcal{O}_{W}\left(2 K_{W}+L\right)\right)=k-4 \geq 0$.

Since $h^{0}\left(V, \mathcal{O}_{V}\left(2 K_{V}\right)\right)=h^{0}\left(S, \mathcal{O}_{S}\left(2 K_{S}\right)\right)=K_{S}^{2}+1$, we conclude that the second summand in (5) is not 0 . For every $i=1, \ldots, k$, one has that

$$
C_{i}\left(2 K_{W}+B_{0}+\sum_{i} C_{i}\right)=-2
$$

and so $\left|2 K_{W}+B_{0}+\sum_{i} C_{i}\right|=\left|2 K_{W}+B_{0}\right|+\sum_{i} C_{i}$. This proves (i).

In Proposition 3.1(i), we already proved that $D$ is nef and big. Therefore $D$ is 1 -connected (see e.g. Lemma 2.6 in [Me]), hence $h^{0}\left(D, \mathcal{O}_{D}\right)=1$, and the final assertion of (ii) follows by the Riemann-Roch Theorem.

Finally for assertion (iii), it suffices to note that, by Propositions 3.1 and 3.3 . $K_{W} D \geq 0$ and $D^{2}>0$.

Corollary 3.5. Let $S$ be a minimal surface of general type with $p_{g}=0$ and $\sigma$ an involution of $S$. Then, with $W$ as above, $K_{W}^{2} \geq K_{V}^{2}$.

Proof. By Noether's formula, because $\chi\left(\mathcal{O}_{W}\right)=\chi\left(\mathcal{O}_{V}\right)$ and $e(W) \leq e(V)$, since the map $\tilde{\pi}$ determines an injection of $H^{2}(W, \mathbb{C})$ into $H^{2}(V, \mathbb{C})$.

Corollary 3.6. Let $S$ be a minimal surface of general type with $p_{g}=0$, let $\varphi$ : $S \rightarrow \mathbb{P}_{S}^{2}$ be the bicanonical map of $S$ and let $\sigma$ be an involution of $S$. Then the following conditions are equivalent:

(i) $\varphi$ is composed with $\sigma$;

(ii) $h^{0}\left(W, \mathcal{O}_{W}\left(2 K_{W}+L\right)\right)=0$;

(iii) $K_{W}\left(K_{W}+L\right)=0$;

(iv) the number of isolated fixed points of $\sigma$ is $k=K_{S}^{2}+4$.

Proof. The bicanonical $\varphi$ is composed with $\sigma$ if and only if one of the summands in formula (5) above vanishes. By Corollary [3.4(i), the second summand is never 0 , and so $\varphi$ is composed with $\sigma$ if and only if $h^{0}\left(W, \mathcal{O}_{W}\left(2 K_{W}+L\right)\right)=0$, which in turn, by Proposition 3.3(iii) and (v), is equivalent to $K_{W}\left(K_{W}+L\right)=0$ and $k=K_{S}^{2}+4$.

Corollary 3.7. Let $S$ be a minimal surface of general type with $p_{g}=0$ and $\sigma$ an involution of $S$. If the bicanonical map $\varphi$ is composed with $\sigma$, then:

(i) $h^{0}\left(W, \mathcal{O}_{W}\left(2 K_{W}+B_{0}\right)\right)=P_{2}(S)=1+K_{S}^{2}$;

(ii) for $D \in\left|2 K_{W}+B_{0}\right|, h^{0}\left(D, \mathcal{O}_{D}(D)\right)=K_{S}^{2}$;

(iii) $K_{W} D=0, D^{2}=2 K_{S}^{2}$ and $p_{a}(D)=K_{S}^{2}+1$;

(iv) $-4 \leq K_{W}^{2} \leq 0$, and $K_{W}^{2}=0$ if and only if $K_{W} \sim 0$;

(v) either $W$ is rational or the minimal model of $W$ is an Enriques surface. 
Proof. Part (i) is immediate from Corollaries 3.4(i) and 3.6(ii). Part (ii) follows from part (i) by considering the long exact sequence obtained from

$$
0 \rightarrow \mathcal{O}_{W} \rightarrow \mathcal{O}_{W}(D) \rightarrow \mathcal{O}_{D}(D) \rightarrow 0
$$

and using that $h^{1}\left(W, \mathcal{O}_{W}\right)=0$. Now note that $D K_{W}=2\left(K_{W}+L\right) K_{W}$, so $K_{W} D=$ 0 follows from Corollary 3.6(iii). Note also that $D^{2}=2 K_{S}^{2}$ by Proposition 3.1(ii), therefore the adjunction formula implies that $p_{a}(D)=K_{S}^{2}+1$. Since $k=K_{S}^{2}+4$ one has that $K_{V}^{2}=-4$, and so the first inequality in (iv) follows from Corollary 3.5. The second inequality and the remainder of statement (iv) follow from the Index Theorem because $D$ is nef and big by Proposition 3.1(i).

Finally, again because $D$ is nef and big, $K_{W} D=0$ implies that $\kappa(W) \leq 0$. So statement (v) follows by the classification of surfaces, since $p_{g}(W)=q(W)=0$.

The following lemma will be used later. We keep the notation introduced above. Namely $D$ is a general member of the non-empty linear system $\left|2 K_{W}+B_{0}\right|$.

Lemma 3.8. Let $S$ be a minimal surface of general type with $p_{g}=0$ and an involution $\sigma$ and let $W$ be as above. If $E \subset W$ is a curve such that $E D=0$, then $E^{2}<0$ and the intersection form on the components of $E$ is negative definite. In particular if $E$ is a curve such that $E^{2}=-1$ and $E D=0$, then $E\left(\sum_{i=1}^{k} C_{i}\right) \leq 0$.

Proof. The first part of the lemma is obvious by the Index Theorem, because $D$ is nef and big by Proposition 3.1(i). For the second part, note that, since $E\left(B_{0}+\sum_{i=1}^{k} C_{i}\right)$ is even and $E D=0, E\left(\sum_{i=1}^{k} C_{i}\right)$ is also even. By the first part applied to the curve $E+C_{i}, i=1, \ldots, k$, one has $E C_{i} \leq 1$. Suppose that $E\left(\sum_{i=1}^{k} C_{i}\right)>0$. Then there would be at least two curves, say $C_{1}$ and $C_{2}$, such that $E C_{1}=E C_{2}=1$, therefore $A=2 E+C_{1}+C_{2}$ would satisfy $A^{2}=0$ and $A D=0$, a contradiction.

Proposition 3.9. Let $S$ be a minimal surface of general type with $p_{g}=0$ and an involution $\sigma$ and let $W$ be as above. Then there exists a birational morphism $f: W \rightarrow W^{\prime}$ and an effective divisor $D^{\prime}$ on $W^{\prime}$ with the following properties:

(i) there are $k(-2)$-curves $C_{i}^{\prime}$ on $W^{\prime}$ such that $f^{*}\left(C_{i}^{\prime}\right)=C_{i}, i=1, \ldots, k$;

(ii) $D^{\prime}$ is nef and such that $f^{*}\left(D^{\prime}\right)=D, D^{\prime 2}=D^{2}$ and $p_{a}\left(D^{\prime}\right)=p_{a}(D)$;

(iii) $K_{W^{\prime}}+D^{\prime}$ is nef.

Proof. If $K_{W}+D$ is nef, there is nothing to prove. Otherwise, since $K_{W}+D$ is effective (see Corollary 3.4), there is an irreducible curve $E_{1}$ such that $E_{1}^{2}<0$ and $E_{1}\left(K_{W}+D\right)<0$. Then $K_{W} E_{1}<0$, thus $E_{1}$ is a $(-1)$-curve and $E_{1} D=0$. Hence $B_{0} E_{1}=2$, and furthermore $E_{1} \cap \bigcup_{i=1}^{k} C_{i}=\emptyset$ by Lemma 3.8.

Let $f_{1}: W \rightarrow W_{1}$ be the contraction of $E_{1}$ to a point $p_{1}$. On $W_{1}$ we have $k(-2)$ curves $C_{i}^{(1)}$ such that $f_{1}^{*}\left(C_{i}^{(1)}\right)=C_{i}, i=1, \ldots, k$, and a nef divisor $D_{1}$ on it such that $D_{1}^{2}=D^{2}$ and $f_{1}^{*}\left(D_{1}\right)=D$. We set $B_{0}^{(1)}=f_{1}\left(B_{0}\right)$. Note that $B_{0}^{(1)}$ has a double point at $p_{1}$, and it does not meet $\bigcup_{i=1}^{k} C_{i}^{(1)}$. Note also that $D_{1} \equiv 2 K_{W_{1}}+B_{0}^{(1)}$.

If $K_{W_{1}}+D_{1}$ is nef, we have finished. Otherwise, reasoning as above, there is a $(-1)$-curve $E_{2}$ on $W_{1}$ such that $E_{2}\left(K_{W_{1}}+D_{1}\right)<0, D_{1} E_{2}=0$ and $E_{2} B_{0}^{(1)}=2$. The curve $E^{\prime}=f_{1}^{*}\left(E_{2}\right)$ satisfies $E^{\prime} D=0, E^{\prime 2}=-1$ and $K_{W} E^{\prime}=-1$ and, since $E_{1}$ is disjoint from the curve $\sum_{i=1}^{k} C_{i}^{(1)}$, none of the curves $C_{i}$ is a component of $E^{\prime}$. Hence, by Lemma 3.8, $E^{\prime} \cap \sum_{i=1}^{k} C_{i}^{(1)}=\emptyset$, implying that $E_{2}$ is also disjoint from the curves $C_{i}^{(1)}$. So we can contract $E_{2}$ and proceed. 
Finally, the existence of the morphism $f: W \rightarrow W^{\prime}$ is shown by iterating the above procedure.

Remark 3.10. We note that, by the proof of Proposition 3.9, the divisor $D^{\prime}+C_{1}^{\prime}+$ $\cdots+C_{k}^{\prime}$ is divisible by 2 in $\operatorname{Pic}\left(W^{\prime}\right)$.

Later, we will need the following lemma, due to Beauville (Lemme 2 in $[\mathrm{Be}]$ ).

Lemma 3.11. Let $\tilde{\pi}: V \rightarrow W$ be a flat double cover between two smooth surfaces $V$ and $W$, branched over the smooth curve $\tilde{B}=B_{1}+\cdots+B_{n}$, where $B_{1}, \ldots, B_{n}$ are the irreducible components of $\tilde{B}$. Suppose that $\operatorname{Pic}(W)$ has no 2-torsion element. Define a group homomorphism $\psi: \mathbb{Z}_{2}^{\oplus n} \rightarrow \operatorname{Pic}(W) \otimes \mathbb{Z}_{2}$ by $\psi\left(\varepsilon_{1}, \ldots, \varepsilon_{n}\right)=\sum_{i=1}^{n} \varepsilon_{i} B_{i}$. Then $\operatorname{Tors}_{2}(V) \cong \operatorname{ker}(\psi) /\langle(1, \ldots, 1)\rangle$.

\section{Numerical Godeaux surfaces With an involution}

In the remainder of this paper, we focus on the study of a numerical Godeaux surface $S$, i.e. a minimal surface of general type with $p_{g}=0$ and $K_{S}^{2}=1$, having an involution $\sigma$. We will freely use the notation introduced so far.

We start by recalling the following.

Theorem 4.1 (Miyaoka, Mi]). Let $S$ be a numerical Godeaux surface. Then the torsion group $\operatorname{Tors}(S)$ of $S$ is cyclic of order $n \leq 5$. Moreover the linear system $\left|3 K_{S}\right|$ has no fixed part and has at most 2 base points. If there is no base point, then $|\operatorname{Tors}(S)| \leq 2$. If there is one base point, then $3 \leq|\operatorname{Tors}(S)| \leq 4$. If there are two base points, then $|\operatorname{Tors}(S)|=5$.

Lemma 4.2 (Lemma 5 in [Mi]). Let $S$ be a numerical Godeaux surface. If $D$ is an effective divisor with $h^{0}(S, D) \geq 2$, then $D K_{S} \geq 2$.

Lemma 4.3 (Reid, see p. 158 in [Do]). Let $S$ be a numerical Godeaux surface. If $\eta$ is a non-trivial element of $\operatorname{Tors}(S)$, then there is a unique element $P_{\eta}$ in $\left|K_{S}+\eta\right|$. Furthermore, if $\eta$ and $\eta^{\prime}, \eta \neq \eta^{\prime}$, are non-trivial elements of $\operatorname{Tors}(S)$, then $P_{\eta}$ and $P_{\eta^{\prime}}$ have no common components.

As an immediate consequence of Lemma 4.3, we have the following.

Corollary 4.4. Let $S$ be a numerical Godeaux surface. If $|\operatorname{Tors}(S)|=4$ or 5 , then $\left|2 K_{S}\right|$ has no fixed components.

Our results in section 3 imply that $S$ enjoys the following properties (cf. also KL $)$.

Proposition 4.5. If $S$ is a numerical Godeaux surface with an involution $\sigma$, then:

(i) the number of isolated fixed points of $\sigma$ is $k=5$;

(ii) the bicanonical map $\varphi$ is composed with $\sigma$;

(iii) $K_{S} R=1$;

(iv) $R^{2}$ is odd and $-7 \leq R^{2} \leq 1$.

Furthermore $R=\Gamma+Z_{1}+\cdots+Z_{h}$ where:

(v) $\Gamma$ is a smooth curve with $K_{S} \Gamma=1,0 \leq p_{a}(\Gamma) \leq 2$ and $\Gamma^{2}=2 p_{a}(\Gamma)-3$;

(vi) if $p_{a}(\Gamma)=2$, then $\Gamma \sim K_{S}$ and $S$ has non-trivial torsion;

(vii) $Z_{1}, \ldots, Z_{h}$ are disjoint $(-2)$-curves, which are disjoint also from $\Gamma$, and

$$
h=p_{a}(\Gamma)-K_{W}^{2}-2 \geq 0 .
$$


Proof. By Proposition 3.3(i) and (v), the number $k$ is odd and $4 \leq k \leq 5$. Hence $k=5$, that is, part (i). Then, part (ii) follows by Corollary 3.6, whilst part (iii) follows from (i) and Lemma 3.2 .

Let us prove part (iv). Since $2 R^{2}=B^{2}$, one has $4 L^{2}=2 R^{2}-10$ and thus $L^{2}=\left(R^{2}-5\right) / 2$. By Proposition 3.3(ii) and Corollary 3.6(iii), $L^{2}=-2-K_{W} L=$ $-2+K_{W}^{2}$, hence $R^{2}=1+2 K_{W}^{2}$. Then by Corollary 3.7 (iv), $-7 \leq R^{2} \leq 1$.

Part (iii) implies that $R$ has a unique irreducible component $\Gamma$ such that $K_{S} \Gamma=$ 1. Since $R$ is smooth, so is $\Gamma$. We can write $R=\Gamma+Z$, where $Z$ is effective. Then $K_{S} Z=0$, thus the irreducible components of $Z$ are $(-2)$-curves, which are pairwise disjoint and disjoint from $\Gamma$ because $R$ is smooth.

By the Index Theorem, $\Gamma^{2} \leq 1$. Since $K_{S} \Gamma=1$, by adjunction one has $\Gamma^{2}=$ $2 p_{a}(\Gamma)-3$, which ends the proof of statement $(\mathrm{v})$.

If $\Gamma^{2}=1$, then $\Gamma$ is homologous to $K_{S}$, but not linearly equivalent to $K_{S}$, because $p_{g}=0$. Therefore $\Gamma-K_{S}$ is a non-trivial torsion element of the Néron-Severi group of $S$, which shows (vi).

Finally (vii) follows from $R^{2}=1+2 K_{W}^{2}=\Gamma^{2}-2 h$ and $\Gamma^{2}=2 p_{a}(\Gamma)-3$.

Remark 4.6. The statements about $R$ on $S$ in Proposition 4.5 can be read as well as about the curve $B_{0}$ on the surface $W$; cf. diagram (1) and Corollary 3.7, For instance, $\tilde{\pi}\left(\epsilon^{-1}\left(Z_{i}\right)\right)$ is a smooth rational curve on $W$ with self-intersection -4 and is an irreducible component of $B_{0}$.

Now Beauville's Lemma 3.11 and Theorem 4.1 imply the following.

Corollary 4.7. Let $h$ be the number of $(-2)$-curves of $R$ as in Proposition 4.5. If $W$ is a rational surface, then

$$
h \leq 1+\left[\frac{-K_{W}^{2}}{2}\right]
$$

and if equality holds, then $S$ has non-trivial 2-torsion.

Proof. Consider the map $\psi$ of Lemma 3.11. The domain of $\psi$ is $\mathbb{Z}_{2}^{\oplus(h+6)}$, because the branch locus of $\tilde{\pi}: V \rightarrow W$ has $h+6$ irreducible components. Moreover the image of $\psi$ is a totally isotropic subspace of $\operatorname{Pic}(W) \otimes \mathbb{Z}_{2}=H^{2}\left(W, \mathbb{Z}_{2}\right)$.

Since $W$ is simply connected, $h^{2}\left(W, \mathbb{Z}_{2}\right)=h^{2}(W, \mathbb{Z})$. Noether's formula implies that $b_{2}(W)=10-K_{W}^{2}$, hence a totally isotropic subspace of $H^{2}\left(W, \mathbb{Z}_{2}\right)$ has dimension at most $5+\left[-K_{W}^{2} / 2\right]$. By Theorem 4.1 , $\operatorname{dim} \operatorname{ker}(\psi) \leq 2$, therefore $h+6 \leq 2+5+\left[-K_{W}^{2} / 2\right]$, which proves (7). If equality holds in (7), then $\operatorname{dim} \operatorname{ker}(\psi)=2$, so $\operatorname{Tors}_{2}(V)$ and $\operatorname{Tors}_{2}(S)$ are not trivial by Lemma 3.11 .

In order to prove the Classification Theorem, stated in the Introduction, we need to understand the surface $W$ and the divisor $D \equiv 2 K_{W}+B_{0}$ on $W$. By Proposition 4.5 and Corollaries 3.4 and 3.7. one has that $D$ is nef, $D^{2}=2, K_{W} D=0, p_{a}(D)=2$, and $h^{0}\left(W, \mathcal{O}_{W}(D)\right)=2$.

If $R$ has a component $\Gamma$ with $p_{a}(\Gamma)=2$, we have further information on $W, D$.

Corollary 4.8. Let $S$ be a numerical Godeaux surface with an involution $\sigma$. Suppose that $R$ has an irreducible component $\Gamma$ of genus 2 . Then either one of the following two cases occurs:

(i) $W$ is a minimal Enriques surface, $R=\Gamma$ and $|D|=\left|B_{0}\right|$;

(ii) $W$ is a rational surface, $-2 \leq K_{W}^{2} \leq-1$ and $\Gamma-K_{S} \in \operatorname{Tors}_{2}(S)$. 
Proof. On $W$, let $\Gamma_{0}=\eta^{*}(\pi(\Gamma)) \leq B_{0}$, thus $\Gamma_{0}^{2}=2$ and $K_{W} \Gamma_{0}=0$. Write

$$
D \equiv 2 K_{W}+B_{0} \equiv 2 K_{W}+\Gamma_{0}+\left(B_{0}-\Gamma_{0}\right) \text {. }
$$

Now $\Gamma_{0} D=\Gamma_{0}^{2}=D^{2}$, so, by the Index Theorem, $\Gamma_{0} \sim D$. Hence (8) implies that $2 K_{W}+B_{0}-\Gamma_{0} \sim 0$. By Corollary 3.7(v), either the minimal model of $W$ is an Enriques surface or $W$ is rational. In the former case $2 K_{W}$ is an effective divisor. Since $B_{0}-\Gamma_{0}$ is also effective, $2 K_{W}+B_{0}-\Gamma_{0} \sim 0$ implies that $0 \equiv 2 K_{W} \equiv B_{0}-\Gamma_{0}$, that is, case (i) of the statement.

If $W$ is rational, numerical equivalence is the same as linear equivalence and so $\Gamma_{0} \equiv D$ and $B_{0}-\Gamma_{0} \equiv-2 K_{W}$, therefore $2 \Gamma \equiv 2 K_{S}$. By formula (6) $B_{0}$ has $1-K_{W}^{2}$ irreducible components, i.e. $h=-K_{W}^{2}$, thus formula (7) implies $K_{W}^{2} \geq-2$.

Remark 4.9. We will see later, in Proposition 7.10, that case (ii) in Corollary 4.8 does not actually occur.

We now study $W$ and the pencil $|D|$ on $W$ by using adjunction.

Lemma 4.10. With the above notation, write $\left|K_{W}+D\right|=F+|M|$, where $F$ denotes the fixed part and $|M|$ the movable part. Then:

(i) $h^{0}\left(W, \mathcal{O}_{W}(M)\right)=2$;

(ii) the general curve of the pencil $|M|$ is irreducible;

(iii) $M D=2$;

(iv) if $F \neq 0$, then every component $E$ of $F$ is such that $D E=0$ and $E^{2}<0$.

Proof. Assertion (i) follows since $p_{a}(D)=2$ and $W$ is regular. Assertion (ii) follows by Bertini's Theorem, since every pencil on the regular surface $W$ is rational.

Let us prove part (iii). Since $D$ is nef and $D(M+F)=2$, one has $M D \leq 2$. Suppose by contradiction that $M D \leq 1$. Consider the pull-back $|\tilde{M}|$ of $|M|$ to $V$, which is also the pull-back of a pencil $|N|$ on $S$. Since $\tilde{\pi}^{*}(D)=\epsilon^{*}\left(2 K_{S}\right)$, one would have that $N K_{S} \leq 1$, which is impossible by Lemma 4.2. This proves (iii).

Assertion (iv) now follows by the Index Theorem.

We have already seen that $-4 \leq K_{W}^{2} \leq 0$. Now we want to consider the surface $W^{\prime}$ as in Proposition 3.9.

Lemma 4.11. One has $-2 \leq K_{W^{\prime}}^{2} \leq 0$. Furthermore $K_{W^{\prime}}^{2}=0$ if and only if $W^{\prime}$ is a minimal Enriques surface.

Proof. Since $D^{\prime}\left(K_{W^{\prime}}+D^{\prime}\right)=2$, the Index Theorem implies that $\left(K_{W^{\prime}}+D^{\prime}\right)^{2} \leq 2$, or equivalently $K_{W^{\prime}}^{2} \leq 0$, because $K_{W^{\prime}} D^{\prime}=0$ and $D^{\prime 2}=2$. On the other hand, $\left(K_{W^{\prime}}+D^{\prime}\right)^{2} \geq 0$ and therefore $K_{W^{\prime}}^{2} \geq-2$.

If $K_{W^{\prime}}^{2}=0$, then $K_{W^{\prime}} \sim 0$, and therefore $W^{\prime}$ is an Enriques surface. The converse is trivial.

Lemma 4.12. If $K_{W^{\prime}}^{2}<0$, then $\left|K_{W^{\prime}}+D^{\prime}\right|=\left|M^{\prime}\right|$ has no fixed part.

Proof. Write, as usual, $\left|K_{W^{\prime}}+D^{\prime}\right|=F^{\prime}+\left|M^{\prime}\right|$, where $F^{\prime}$ is the fixed part and $\left|M^{\prime}\right|$ is the movable part. By Lemma 4.10(iii), and the construction of the morphism $f: W \rightarrow W^{\prime}$, which contracts only curves in $F$, we see that $M^{\prime} D^{\prime}=2$.

Note that $F^{\prime} K_{W^{\prime}}=F^{\prime} M^{\prime}+F^{\prime 2}$, so $M^{\prime} F^{\prime}$ is even. Since $M^{\prime} F^{\prime} \leq M^{\prime} F^{\prime}+M^{\prime 2} \leq$ $\left(K_{W^{\prime}}+D^{\prime}\right)^{2}=K_{W^{\prime}}^{2}+2<2$, it follows that $M^{\prime} F^{\prime}=0$. This forces $F^{\prime}$ to be 0 . Otherwise, since $D^{\prime} F^{\prime}=0$, then $F^{2}<0$, implying that $F^{\prime}\left(K_{W^{\prime}}+D^{\prime}\right)=$ $F^{\prime 2}+M^{\prime} F^{\prime}<0$, a contradiction because $\left(K_{W^{\prime}}+D^{\prime}\right)$ is nef. 
Remark 4.13. Since $K_{W}^{2} \geq-4$ by Corollary 3.7(iv), the birational map $f: W \rightarrow W^{\prime}$ of Proposition 3.9 is the contraction of at most 3 (resp., at most 4) exceptional curves if $W^{\prime}$ is rational (resp., an Enriques surface).

Note that an unessential singularity of $B_{0}^{\prime}$ corresponding to a triple point requires at least 4 blowing-ups to be resolved, and exactly 4 unless there is a double point infinitely near to the triple point. Thus $B_{0}^{\prime}$ can have a triple point only if $W^{\prime}$ is a minimal Enriques surface, $K_{W}^{2}=-4$ and $B_{0}^{\prime}$ has only one triple point with no infinitely near double point.

If $K_{W}^{2}=-4$, then $\tilde{\pi}$ induces an isomorphism between $H^{2}(W, \mathbb{Z})$ and $H^{2}(V, \mathbb{Z})$. This implies that there is no rational curve on $W$ that does not meet the branch locus $\tilde{B}$. As a consequence, an unessential singularity of $B_{0}^{\prime}$ can only be an ordinary triple point, a node or a cusp.

\section{On a numerical Godeaux surface of Enriques type}

In this section we keep the notation we introduced in the above sections.

Let $S$ be a numerical Godeaux surface with an involution. Here we analyze the case in which $W$ is birational to an Enriques surface (see Corollary 3.7(v)). This situation corresponds to the case in which $W^{\prime}$ is a minimal Enriques surface and $B_{0}^{\prime}=D^{\prime}$ (see Lemma 4.11). The main information is given by the following result.

Proposition 5.1. In the above setting, the general curve in the linear system $\left|D^{\prime}\right|$ is smooth and irreducible.

Proof. Since $D^{\prime 2}=2$ and $h^{0}\left(W^{\prime}, \mathcal{O}_{W^{\prime}}\left(D^{\prime}\right)\right)=2$ by Lemma 4.10 and Proposition 3.9, it suffices to prove that the linear system $\left|D^{\prime}\right|$ has no fixed component. Assume the contrary and write $\left|D^{\prime}\right|=\Phi+|\Psi|$, where $\Phi$ is the fixed part. Since $D^{\prime}$ is nef, then $D^{\prime} \Psi \leq 2$. On the other hand, by Lemma 4.2 we have $D^{\prime} \Psi=2$. This implies $\Psi^{2}=0, \Psi \Phi=2$, and $\Phi^{2}=-2$. Moreover by Proposition 3.9. one has $B_{0}^{\prime} \cap\left(C_{1}^{\prime}+\cdots+C_{5}^{\prime}\right)=\emptyset$, which implies $\Psi\left(C_{1}^{\prime}+\cdots+C_{5}^{\prime}\right)=0$. Now we remark that $|\Psi|$ is a pencil of elliptic curves on the Enriques surface $W^{\prime}$, thus $\Psi$ is divisible by 2 in $\operatorname{Pic}\left(W^{\prime}\right)$. Since $\Psi+\Phi+C_{1}^{\prime}+\cdots+C_{5}^{\prime}$ is also divisible by 2 , we have that $\Phi+C_{1}^{\prime}+\cdots+C_{5}^{\prime}$ is divisible by 2 . Since $\Psi\left(\Phi+C_{1}^{\prime}+\cdots+C_{5}^{\prime}\right)=\Psi \Phi=2$, we find a contradiction.

Remark 5.2. The above proposition also tells us that every numerical Godeaux surface with an involution of Enriques type is deformation equivalent to the double cover of a minimal Enriques surface $W$ with five nodes $C_{1}, \ldots, C_{5}$, branched along a smooth curve $B_{0}+C_{1}+\cdots+C_{5}$, where $B_{0}$ is irreducible of genus 2 . There are examples of surfaces of this type (see Example 4.3 of [KL]; cf. Corollary 4.8 and Proposition 7.10 below).

Next we describe the torsion group of these surfaces.

Proposition 5.3. The torsion group of a numerical Godeaux surface $S$ of Enriques type is $\mathbb{Z}_{4}$.

Proof. By Remark 5.2, we may assume that $W$ is a minimal Enriques surface and that $B_{0}$ is a smooth, irreducible curve of genus 2. One has a cartesian diagram

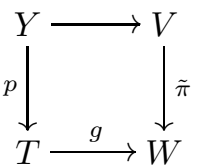


where $g: T \rightarrow W$ is the K3-double cover of $W$, so $Y \rightarrow V$ is an étale double cover, where the minimal model of $Y$ has $K^{2}=2$ and $p_{g}=1$. Thus $V$, and hence $S$, has 2-torsion. Moreover $\operatorname{Tors}(V)=\mathbb{Z}_{4}$ if and only if $\operatorname{Tors}(Y)=\mathbb{Z}_{2}$.

Let us now look at the double cover $p: Y \rightarrow T$ which is branched along the curve $H:=g^{*}\left(B_{0}\right)$ and the ten $(-2)$-curves which are the pull-back via $g$ of the five (-2)curves $C_{1}, \ldots, C_{5}$. Standard double cover considerations show that the bicanonical map of $Y$ factors through $p$, in particular it is not birational. By Theorem 6.1 of $\mathrm{CaD}$, this implies that $Y$ has 2-torsion, which implies the assertion.

Remark 5.4. By Proposition 5.3 and Corollary 4.4, it follows that, if $S$ is a numerical Godeaux surface of Enriques type, then $\left|2 K_{S}\right|$ has no fixed component.

\section{On numerical Godeaux surfaces of Campedelli type}

In this section we follow the notation introduced above and we study the case $K_{W^{\prime}}^{2}=-2$ (cf. Lemma 4.11). As we will see, this case corresponds to numerical Godeaux surfaces which are birationally equivalent to double planes branched along a curve of degree 10 with a point of multiplicity 4 and five points of type [3,3]. As we said in the Introduction, we call these surfaces of Campedelli type.

Lemma 6.1. Let $S$ be a numerical Godeaux surface with an involution and let $W^{\prime}$ be as in sections 3, 4. Suppose that $K_{W^{\prime}}^{2}=-2$. Then $\left|M^{\prime}\right|$ is a base point free pencil of rational curves. Furthermore, if $A$ is an irreducible component of a reducible fibre of $\left|M^{\prime}\right|$, then one of the following occurs:

(i) either $A$ is a (-1)-curve such that $D^{\prime} A=1$ and $B_{0}^{\prime} A=3$;

(ii) or $A$ is a (-2)-curve such that $D^{\prime} A=B_{0}^{\prime} A=0$.

Proof. The hypothesis $K_{W^{\prime}}^{2}=-2$ implies that $M^{\prime 2}=0$ and $K_{W^{\prime}} M^{\prime}=-2$, so that $\left|M^{\prime}\right|$ is a base point free pencil of rational curves.

An irreducible component of a reducible fibre of $\left|M^{\prime}\right|$ is a rational curve $A$ with $A^{2}<0$. Statements (i) and (ii) follow from $0=M^{\prime} A=\left(K_{W^{\prime}}+D^{\prime}\right) A \geq K_{W^{\prime}} A=$ $-2-A^{2}$, where the inequality holds because $D^{\prime}$ is nef.

Lemma 6.1] immediately implies the following corollary (cf. also [Xi], [Ho]).

Corollary 6.2. In the above setting, if $K_{W^{\prime}}^{2}=-2$, a reducible fibre of $\left|M^{\prime}\right|$ is of one of the following types:

(i) either it contains two irreducible (-1)-curves, in which case the dual graph of the fibre is a chain as in Figure 1(i);

(ii) or it contains only one (-1)-curve with multiplicity 2 , in which case the dual graph of the fibre is shown in Figure 1(ii) and (ii').

In Figure 1 the black (resp. white) vertices correspond to curves contained (resp. not contained) in the branch locus.

Remark 6.3. Note that the number of the vertices in the graphs in Figure 1 is always odd. For every $i=1, \ldots, 5$, one has $C_{i}^{\prime} M^{\prime}=C_{i}^{\prime}\left(K_{W^{\prime}}+D^{\prime}\right)=0$, thus the $(-2)$-curve $C_{i}^{\prime}$ is contained in a curve of the pencil $\left|M^{\prime}\right|$. It is easy to verify (cf. also [Ho] ) that the black vertices in Figure 1 correspond to the curves $C_{1}^{\prime}, \ldots, C_{5}^{\prime}$, except one of the $(-2)$-curves in $\left(\right.$ ii $\left.^{\prime}\right)$, which is an irreducible component of $B_{0}^{\prime}$.

Since $M^{\prime} D^{\prime}=2$, the pencil $(f \circ \tilde{\pi})^{*}\left(\left|M^{\prime}\right|\right)$ is a genus 2 pencil without base points on $V$ which descends via $\epsilon$ to a genus 2 pencil without base points on $S$. 


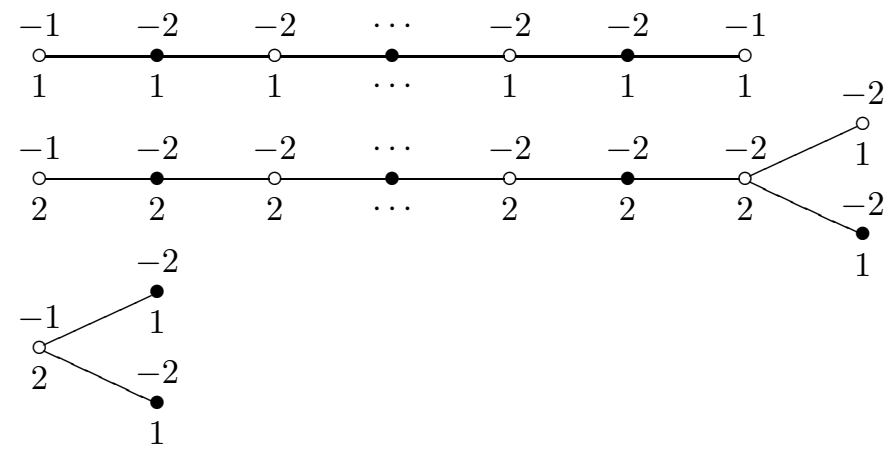

FiguRE 1. Reducible fibres of $M^{\prime}$ with self-intersections and multiplicities.

Proposition 6.4. In the above setting, if $K_{W^{\prime}}^{2}=-2$, then there exists a birational morphism $g: W^{\prime} \rightarrow \mathbb{F}_{a}$ with either $a=1$ or $a=3$. Let $\Delta$ be the exceptional divisor of $g$, let $E$ be the $(-a)$-curve on $\mathbb{F}_{a}$ and let $\Theta=g^{*}(E)$ be its total transform on $W^{\prime}$. Then

$$
D^{\prime} \equiv 2 \Theta+(a+3) M^{\prime}-\Delta .
$$

Furthermore, if $a=3$, then $\Theta$ is also the proper transform of $E$ on $W^{\prime}$.

Proof. As seen in Remark 6.3. each (-2)-curve $C_{i}^{\prime}$ is contained in a (reducible) curve of the pencil $\left|M^{\prime}\right|$.

We claim that there exists a birational morphism $g: W^{\prime} \rightarrow \mathbb{F}_{a}$, for some $a \geq 0$, which contracts each of the curves $C_{i}^{\prime}$ to points. Suppose that the fibre to which $C_{i}^{\prime}$ belongs is of type (i) of Corollary 6.2 In a birational morphism of $W^{\prime}$ to a $\mathbb{F}_{a}$, every component of such a fibre is contracted to a point, except one of the components corresponding to an end-point. This proves our claim in this case.

Otherwise, the fibre is of type either (ii) or (ii') of Corollary 6.2. In a birational morphism of $W^{\prime}$ to a $\mathbb{F}_{a}$, every component of such a fibre is contracted to a point, except either one of the two (-2)-components of multiplicity 1, corresponding to one of the two right end-points of the graphs in Figure 1, In both cases, there is only one of the $(-2)$-components which is one of the $C_{i}^{\prime}$ 's (cf. Remark 6.3), thus we may and will choose to contract it. This concludes the proof of our claim.

Recall that, since $K_{\mathbb{F}_{a}} \equiv-2 E-(a+2) F$, where $F$ is the ruling of $\mathbb{F}_{a}, K_{W^{\prime}} \equiv$ $-2 \Theta-(a+2) M^{\prime}+\Delta$. Then formula (10) follows from $K_{W^{\prime}}+D^{\prime} \equiv M^{\prime}$.

Since $D^{\prime}$ is nef, one has that $0 \leq D^{\prime} \Theta=-2 a+a+3$ by (10), hence $a \leq 3$.

Let $\bar{\Theta}$ be the proper transform of $E$ on $W^{\prime}$. Again, since $D^{\prime}$ is nef, one has

$$
0 \leq D^{\prime} \bar{\Theta}=-2 a+a+3-\Delta \bar{\Theta}=-a+3-\Delta \bar{\Theta} .
$$

In particular, if $a=3$, it follows that $\Delta \bar{\Theta}=0$, which means that $\bar{\Theta}=\Theta$.

It remains to prove that $a$ is odd. Note that $g$ induces a double cover $\bar{\pi}: \bar{V} \rightarrow \mathbb{F}_{a}$ branched along the curve $\bar{B}_{0}=g\left(B_{0}^{\prime}\right)$, which has to be an even divisor in $\operatorname{Pic}\left(\mathbb{F}_{a}\right)$. Since

$$
B_{0}^{\prime} \equiv D^{\prime}-2 K_{W^{\prime}} \equiv 6 \Theta+(3 a+7) M^{\prime}-3 \Delta,
$$

then $\bar{B}_{0} \equiv 6 E+(3 a+7) F$, which shows that $a$ has to be odd. 
Remark 6.5. Passing from $W^{\prime}$ to $\mathbb{F}_{a}$ creates only essential singularities of the branch curve $\bar{B}_{0}$ of the double cover $\bar{\pi}: \bar{V} \rightarrow \mathbb{F}_{a}$. More precisely, a fibre of type (i), as in Figure 1, with $2 l+1$ vertices produces $2 l+1$ a point $x$ of type $\left[3^{2 l}\right]$, whose tangent direction is different from the tangent direction of the fibre $F_{x}$ of $\mathbb{F}_{a}$ through $x$. Here we denote by $\left[3^{n}\right]$ the sequence $[3, \ldots, 3]$ of length $n$, so a point of type $\left[3^{n}\right]$ is a triple point with an infinitely near point of type $\left[3^{n-1}\right]$.

Instead, as in Figure 1(ii) and (ii'), a fibre with $2 l+1$ vertices again produces a point $x$ of type $\left[3^{2 l}\right]$, but the tangent direction coincides with the one of $F_{x}$. In case (ii') (resp. case (ii)), the fibre $F_{x}$ is (resp. is not) a component of $\bar{B}_{0}$.

Now we show that the case $a=3$ can be reduced to the case $a=1$.

Lemma 6.6. If $a=3$, the branch curve $\bar{B}_{0}$ of the double cover $\bar{\pi}: \bar{V} \rightarrow \mathbb{F}_{3}$ is of the form $\bar{B}_{0}=E+\bar{B}$, where $E$ is the $(-3)$-curve in $\mathbb{F}_{3}$ and $\bar{B} E=1$. Furthermore, there is a birational map $h: \mathbb{F}_{3} \rightarrow \mathbb{F}_{1}$ such that $h \circ g: W^{\prime} \rightarrow \mathbb{F}_{1}$ is a birational morphism contracting all the $(-2)$-curves $C_{i}^{\prime}, i=1, \ldots, 5$, to points as in Proposition 6.4.

Proof. Formula (10) says that $\bar{B}_{0} \equiv 6 E+16 F$, which implies the first assertion.

By Remark $6.5, \bar{B}$ has five points of type $[3,3]$, which are off $E$, because $E \bar{B}=1$, and can be proper or infinitely near. Let $x$ be a [3,3]-point of $\bar{B}_{0}$ and let $F_{x}$ be the fibre of the ruling of $\mathbb{F}_{3}$ through $x$. Since $\bar{B} F_{x}=5$, Remark 6.5 again implies that the fibre $F_{x}$ is not of type (ii) as in Figure 1. If $F_{x}$ is of type (ii'), then $F_{x}$ is a component of $\bar{B}$ and $\bar{B} E=F_{x} E=1$, thus there is at most one such fibre. Therefore there are fibres of type (i), and we may choose a point $x \in \mathbb{F}_{3}$ such that $\bar{B}$ has a [3,3]-point at $x$, whose tangent direction is different from the one of the fibre $F_{x}$ through $x$ (and $F_{x}$ is not a component of $\bar{B}$ ).

Now we define the birational map $h: \mathbb{F}_{3} \rightarrow \mathbb{F}_{1}$ as the composition of two elementary transformations:

- the first one is based at the point $x$. This leads to $\mathbb{F}_{2}$, and the proper transform of $\bar{B}$ shows the triple point $y$ which was infinitely near to $x$;

- the second elementary transformation is based at $y$.

The birational map $h \circ g$ is a morphism because the exceptional curves created by the two elementary transformations are contracted by $g$, by construction.

Remark 6.7. In the proof of the above lemma, we saw that, if $a=3$, then there is no fibre of type (ii), and at most one fibre of type (ii').

Since $\bar{B}_{0}$ has a double point where $\bar{B}$ meets $E$, then $\bar{B}$ has at most one unessential singularity, which is resolved by one blowing-up (cf. Remark 4.13).

Finally we deal with the case $a=1$ of Proposition 6.4

Lemma 6.8. In the above setting, if $a=1$ and $E$ is a component of the branch locus $\bar{B}_{0}$ of $\bar{\pi}: \bar{V} \rightarrow \mathbb{F}_{1}$, then $\bar{B}_{0}=E+\bar{B}$, where $\bar{B}$ has a tacnode at a point $x \in E$ where the tangent direction is also tangent to $E$ at $x$. Moreover $\bar{B}$ meets $E$ transversally at another point $y \neq x$.

Proof. By formula (12), one has $E \bar{B}=5$. Since $\bar{B}_{0}$ has at most two unessential singularities (cf. Remark 4.13), then a [3,3]-point of $\bar{B}_{0}$ must lie on $E$.

Now let $g^{\prime}: \mathbb{F}_{1} \rightarrow \mathbb{P}^{2}$ be the contraction of the $(-1)$-curve $E$ of $\mathbb{F}_{1}$ to a point $p \in \mathbb{P}^{2}$. This induces a double plane $\pi^{\bullet}: V^{\bullet} \rightarrow \mathbb{P}^{2}$ branched along $B_{0}^{\bullet}=g^{\prime}\left(\bar{B}_{0}\right)$.

The following corollary proves the Classification Theorem in the case $K_{W^{\prime}}^{2}=-2$. 
Corollary 6.9. Let $S$ be a numerical Godeaux surface with an involution and suppose that $K_{W^{\prime}}^{2}=-2$. Then the double plane $\pi^{\bullet}: V^{\bullet} \rightarrow \mathbb{P}^{2}$, constructed as above, is branched along a reduced curve $B_{0}^{\bullet}$ of degree 10 with a point $q$ of multiplicity at least 4 and five points of type [3,3], which can be distinct or infinitely near, not lying on, a conic, and possibly further unessential singularities resolved by at most two blowing-ups.

Proof. The curve $B_{0}^{\bullet}$ has degree 10 by (12) and multiplicity either 5 or 4 at $q$, depending on whether $E$ is or is not a component of $\bar{B}_{0}^{\prime}$. By Remark 6.5, $B_{0}^{\bullet}$ has five [3, 3]-points, which can be proper or infinitely near. The unessential singularities of $B_{0}^{\bullet}$ are at most two, because $K_{W^{\prime}}^{2}-K_{W}^{2}=-2-K_{W}^{2} \leq 2$ (cf. Remark 4.13).

The fact that there is no conic through $q$ and the five [3,3]-points follows by standard double plane considerations, since $p_{g}(S)=0$.

Remark 6.10. Lemma 6.8 completely describes the nature of the singularity at $q$ in the case where $B^{\bullet}$ has a point of multiplicity 5 at $q$ (cf. also Example 13.3 of [CF]).

Remark 6.11. Note that, if $B^{\bullet}$ is irreducible, then its geometric genus is 0. Examples of this type can be found in [Re2, [St, $\mathrm{CG}$. (as shown by [DW]).

\section{On numerical Godeaux surfaces of Du Val type}

In this section we go on using the notation introduced above, and we study the remaining case $K_{W^{\prime}}^{2}=-1$ (cf. Lemma 4.11). We will show that it gives rise to numerical Godeaux surfaces which are birationally equivalent to double planes branched along a curve of degree 14 as in case (2) of the Classification Theorem, stated in the Introduction.

According to the terminology introduced in Example 3.7(c) of Ci1, this double plane is a degeneration of a Du Val ancestor with invariants $p_{g}=4$ and $K^{2}=8$. As we said in the Introduction, we will call these surfaces of Du Val type.

We start by studying the linear systems $\left|M^{\prime}\right|$ and $\left|K_{W^{\prime}}+M^{\prime}\right|$. Recall that by definition $\left|M^{\prime}\right|=\left|K_{W^{\prime}}+D^{\prime}\right|$ and $\left|M^{\prime}\right|$ has no fixed part by Lemma 4.12

Lemma 7.1. Let $S$ be a numerical Godeaux surface with an involution and let $W^{\prime}$ be as in sections 3, 4. Suppose that $K_{W^{\prime}}^{2}=-1$. Then:

(i) $\left|M^{\prime}\right|$ is a pencil of elliptic curves with a simple base point;

(ii) $h^{0}\left(W^{\prime}, \mathcal{O}_{W^{\prime}}\left(K_{W^{\prime}}+M^{\prime}\right)\right)=1$;

(iii) if $G$ is the unique curve in $\left|K_{W^{\prime}}+M^{\prime}\right|$, then $G M^{\prime}=0$ and $G=2 E+C_{5}^{\prime}$, where $E$ is a $(-1)$-curve and $E C_{5}^{\prime}=E D^{\prime}=1$;

(iv) the divisor $C_{1}^{\prime}+\cdots+C_{4}^{\prime}$ is even in $\operatorname{Pic}\left(W^{\prime}\right)$.

Proof. Since $K_{W^{\prime}}^{2}=-1$, one has $M^{\prime 2}=\left(K_{W}^{\prime}+D^{\prime}\right)^{2}=1$. Moreover $K_{W^{\prime}} M^{\prime}=-1$. This proves (i). The long exact sequence obtained from

$$
0 \rightarrow \mathcal{O}_{W^{\prime}}\left(K_{W^{\prime}}\right) \rightarrow \mathcal{O}_{W^{\prime}}\left(K_{W^{\prime}}+M^{\prime}\right) \rightarrow \mathcal{O}_{M^{\prime}} \rightarrow 0
$$

implies part (ii), because $W^{\prime}$ is rational.

Let us prove part (iii). Since $p_{a}\left(M^{\prime}\right)=0$, one has that $M^{\prime} G=0$, and every component $\theta$ of $G$ is such that $M^{\prime} \theta=0$, because $M^{\prime}$ is nef. Furthermore $M^{\prime} G=0$ implies that the intersection form on the components of $G$ is negative definite. Since $G^{2}=-2$, there is an irreducible curve $E$ in $G$ such that $E^{2}<0$ and $E\left(K_{W^{\prime}}+M^{\prime}\right)=$ $E G<0$. From $M^{\prime} E=0$, we conclude that $E K_{W^{\prime}}<0$, thus $E$ is a $(-1)-$ curve and $E D^{\prime}=1, E G=-1$. Furthermore $E(G-E)=0$. 
We claim that $E$ intersects one of the $(-2)$-curves $C_{i}^{\prime}$, say $C_{5}^{\prime}$. Indeed, since $D^{\prime}+C_{1}^{\prime}+\cdots+C_{5}^{\prime}$ is divisible by 2 by Remark 3.10 , the claim follows, because $E D^{\prime}=1$.

Note that $C_{5}^{\prime} G=C_{5}^{\prime}\left(2 K_{W^{\prime}}+D^{\prime}\right)=0$. Since $E C_{5}^{\prime}>0$, we have $C_{5}^{\prime} \leq G-E$. Since $E(G-E)=0$, then $E$ is necessarily a component of $G-E$. Note that $\left(2 E+C_{5}^{\prime}\right)^{2}=4 E^{2}+4 E C_{5}^{\prime}+C_{5}^{\prime 2}=4 E C_{5}^{\prime}-6$ and so, because the intersection form on the components of $G$ is negative definite, we conclude that $\left(2 E+C_{5}^{\prime}\right)^{2}=-2$. Since $\left(2 E+C_{5}^{\prime}\right) G=-2$, we have $\left(G-\left(2 E+C_{5}^{\prime}\right)\right)^{2}=0$ and, so again by negative definiteness, we see that $G=2 E+C_{5}^{\prime}$.

So the proof of part (iii) is concluded.

Finally we prove part (iv). Since $D^{\prime}+C_{1}^{\prime}+\cdots+C_{5}^{\prime}$ is divisible by 2 , then $2 K_{W^{\prime}}+D^{\prime}+C_{1}^{\prime}+\cdots+C_{5}^{\prime} \equiv C_{5}^{\prime}+2 E+C_{1}^{\prime}+\cdots+C_{5}^{\prime}$ is divisible by 2 , and therefore $C_{1}^{\prime}+\cdots+C_{4}^{\prime}$ is also divisible by 2 .

Remark 7.2. Note that $C_{1}+\ldots+C_{4}$ is an even divisor in $W$ by the previous lemma, hence $B_{0}+C_{5}$ is even, too. So, by Beauville's Lemma 3.11. $\operatorname{Tors}_{2}(S)$ is not trivial and thus, by Theorem 4.1, Tors $(S)$ is either $\mathbb{Z}_{2}$ or $\mathbb{Z}_{4}$.

We will later need the following.

Corollary 7.3. In the above setting, the curve $B_{0}^{\prime}$ is 1-connected.

Proof. Suppose that $B_{0}^{\prime}$ is not 1-connected and write $B_{0}^{\prime}=G+H$ with $G H=0$. Then say $D^{\prime} G=0$ and $D^{\prime} H=2$. Since $D^{\prime}$ is nef, $G^{2}<0$, and therefore $0=$ $D^{\prime} G=2 K_{W^{\prime}} G+G H+G^{2}=2 K_{W^{\prime}} G+G^{2}$ implies that $K_{W^{\prime}} G>0$. Also we note that $H^{2} \leq 1$. In fact the Index Theorem applied to $D^{\prime}$ and $D^{\prime}-H$ implies that $H^{2} \leq 2$ and that $H^{2}=2$ if and only if $D^{\prime} \equiv H$ (because $W^{\prime}$ is rational). But if $D^{\prime} \equiv H$, then $G \equiv-2 K_{W^{\prime}}$ and, since also $C_{1}+\cdots+C_{4}$ is even, this would imply by Beauville's Lemma 3.11 that $\left|\operatorname{Tors}_{2}(S)\right| \geq 2$, contradicting Theorem 4.1. Then $2=D^{\prime} H=2 K_{W^{\prime}} H+H^{2}$ also implies that $K_{W^{\prime}} H>0$. Since $K_{W^{\prime}} B_{0}^{\prime}=2$, it follows that $K_{W^{\prime}} H=K_{W^{\prime}} G=1$. From $0=D^{\prime} G=2 K_{W^{\prime}} G+G^{2}$, we conclude that $G^{2}=-2$, which contradicts the adjunction formula.

By Lemma 7.1, one has that

$$
K_{W^{\prime}} \equiv-M^{\prime}+2 E+C_{5}^{\prime}
$$

Furthermore, since $C_{1}^{\prime}+\cdots+C_{4}^{\prime}$ is an even divisor, then also $D^{\prime}-C_{5}^{\prime} \equiv 2 \Delta$, where $\Delta^{2}=0$ and $K_{W^{\prime}} \Delta=0$. By the Riemann-Roch Theorem, $|\Delta| \neq \emptyset$. The following identities are easy to check:

$$
\begin{aligned}
& M^{\prime} \equiv \Delta+E+C_{5}^{\prime}, \\
& B_{0}^{\prime} \equiv 4 \Delta-2 E+C_{5}^{\prime} \equiv 4 M^{\prime}-6 E-3 C_{5}^{\prime}, \\
& K_{W^{\prime}}+\Delta \equiv E .
\end{aligned}
$$

Remark 7.4. Going back to our original surface $W$, the above formulas mean that $D \equiv 2 \tilde{\Delta}+C_{5}$, where $\tilde{\Delta}$ is the pull-back of $\Delta$ to $W$.

Note that there is a birational morphism $g: W^{\prime} \rightarrow X$ which contracts $G=$ $2 E+C_{5}^{\prime}$ to a smooth point $q$. The rational surface $X$ is such that $K_{X}^{2}=1$ and $g^{*}\left(K_{X}\right) \equiv-M^{\prime}$. Therefore $-K_{X}$ is nef and big.

We denote by $\mathcal{D}$ the pencil $g_{*}\left(\left|D^{\prime}\right|\right)$ and by $D^{\prime \prime} \in \mathcal{D}$ its general element. Then $D^{\prime \prime 2}=4, K_{X} D^{\prime \prime}=-2$ and $\mathcal{D}$ has at $q$ a base point with a fixed tangent. Furthermore $D^{\prime \prime} \equiv-2 K_{X}$. 
We note that $X$ still contains an even set of four disjoint $(-2)$-curves. Now we can apply the following result from [CCM], regarding rational surfaces with an even set of nodes (cf. also $\S 4$ in Chapter 0 of $\mathrm{CoD}$ ).

Theorem 7.5. Let $X$ be a weak Del Pezzo surface, i.e. $-K_{X}$ is big and nef. Assume that $K_{X}^{2}=1$ and that $X$ has four disjoint $(-2)$-curves $C_{1}^{\prime \prime}, \ldots, C_{4}^{\prime \prime}$ such that $C_{1}^{\prime \prime}+\cdots+C_{4}^{\prime \prime}$ is even in $\operatorname{Pic}(X)$. Then there exists a birational morphism $h: X \rightarrow X^{\prime}$, where $X^{\prime}$ is obtained from $\mathbb{F}_{a}$, with $a=0$, 1 , or 2 , by blowing up:

- two points $q_{1}^{\prime}, q_{2}^{\prime}$ in distinct fibres $F_{1}, F_{2}$ of the same ruling of $\mathbb{F}_{a}$;

- the point which is the intersection of the strict transform of $F_{i}$ with the exceptional curve corresponding to $q_{i}, i=1,2$;

in case $a=2$, none of the blown-up points lies on the $(-2)$-curve of $\mathbb{F}_{2}$. The morphism $h$ maps isomorphically the $(-2)$-curves $C_{1}^{\prime \prime}, \ldots, C_{4}^{\prime \prime}$ onto the proper transforms in $X^{\prime}$ of $F_{1}, F_{2}$ and of the exceptional curves corresponding to $q_{1}^{\prime}$ and $q_{2}^{\prime}$.

Next corollary proves the Classification Theorem in the last case (2).

Corollary 7.6. Let $S$ be a numerical Godeaux surface with an involution and suppose that $K_{W^{\prime}}^{2}=-1$. Then there exists a birational morphism $g^{\prime}: W^{\prime} \rightarrow \mathbb{P}^{2}$ such that the induced double cover $\pi^{\bullet}: V^{\bullet} \rightarrow \mathbb{P}^{2}$ is a double plane of Du Val type, branched along a reduced curve $B^{\bullet}$, which possibly has irrelevant singularities, resolved with at most three blowing-ups.

Proof. Suppose that $a=1$ in the statement of Theorem 7.5 i.e. there exists a birational morphism $X \rightarrow \mathbb{F}_{1}$. By blowing-down the (-1)-curve of $\mathbb{F}_{1}$, it determines a birational morphism $h^{\prime}: X \rightarrow \mathbb{P}^{2}$ and hence a birational morphism $g^{\prime}=h^{\prime} \circ g \circ f$ : $W \rightarrow \mathbb{P}^{2}$. Up to reordering the indices, the $(-2)$-curves $C_{1}$ and $C_{2}$ are mapped via $g^{\prime}$ to two distinct lines, say $r_{1}$ and $r_{2}$, whilst $C_{3}$ and $C_{4}$ are contracted to two distinct points $q_{1} \in r_{1}$ and $q_{2} \in r_{2}$. The anti-canonical pencil $\left|-K_{X}\right|$ on $X$ is mapped via $h^{\prime}$ to a pencil of plane cubics with the following eight base points:

- $q_{0}=r_{1} \cap r_{2}$

- $q_{i}, i=1,2$, where the cubics are tangent to $r_{i}$ at $q_{i}$;

- three further simple base points $q_{3}, q_{4}, q_{5}$.

Therefore the pencil $|D|$ on $W$ is mapped via $g^{\prime}$ to a pencil of plane sextics with the following base points:

- double points at $q_{0}, q_{3}, q_{4}, q_{5}$;

- a tacnode at $q_{i}, i=1,2$, where the tacnodal tangent is $r_{i}$;

- a further simple base point $q_{6}$ with fixed tangent.

Standard double plane considerations imply the assertion in this case $a=1$.

Suppose that $a=2$ in the statement of Theorem 7.5, i.e. there exist birational morphisms $X \rightarrow X^{\prime} \rightarrow \mathbb{F}_{2}$. Note that on $X^{\prime}$ there is a (-2)-curve $N$, which is the proper transform of the $(-2)$-curve on $\mathbb{F}_{2}$, and two reducible fibres of type $A_{1}+A_{3}+2 N_{1}$ and $A_{2}+A_{4}+N_{2}$, where $A_{1}, \ldots, A_{4}$ are $(-2)$-curves and $N_{1}, N_{2}$ are (-1)-curves with $N A_{1}=N A_{2}=1, N A_{3}=N A_{4}=N N_{1}=N N_{2}=0$. By first blowing-down $N_{1}, N_{2}$ and then the image of $A_{1}$ and of $A_{4}$, one arrives at $\mathbb{F}_{1}$, and the image of $N$ is the (-1)-curve. At this point, one proceeds as in the previous case. Note that, in the present situation, either $q_{1}$ or $q_{2}$ is infinitely near to $q_{0}$.

Suppose finally that $a=0$. On $X^{\prime}$ there are two reducible fibres of the same ruling of the type $A_{1}+A_{3}+2 N_{1}$ and $A_{2}+A_{4}+2 N_{2}$, where $A_{1}, \ldots, A_{4}$ are (-2)curves and $N_{1}, N_{2}$ are $(-1)$-curves such that, if $N_{0}$ is a general fibre of the other 
ruling, $N_{0} A_{1}=N_{0} A_{2}=1, N_{0} A_{3}=N_{0} A_{4}=N_{0} N_{1}=N_{0} N_{2}=0$. By first blowingdown $N_{1}, N_{2}$ and then the image of $A_{1}$ and of $A_{4}$, one arrives at $\mathbb{F}_{1}$, because the image of $N_{0}$ is a section with self-intersection 1 , and one concludes as before.

Remark 7.7. Note that, if the degree 12 component of $B^{\bullet}$ is irreducible, then its geometric genus is 1 .

Remark also that all irrelevant singularities are double points, because four blowing-ups are needed to resolve an irrelevant triple point (cf. Remark 4.13).

Remark 7.8. The morphism $g^{\prime}: W \rightarrow \mathbb{P}^{2}$ defined in the proof of Corollary 7.6 maps the curve $\tilde{\Delta}$ of Remark 7.4 to a plane cubic $\bar{\Delta}$. Indeed the curve $\Delta$ on $W^{\prime}$ (cf. formulas (13)-(16)) is mapped to $\bar{\Delta}$ via $h^{\prime} \circ g: W^{\prime} \rightarrow \mathbb{P}^{2}$.

Remark 7.9. Consider $X^{\prime}$ as in Theorem 7.5. Let $\bar{C}_{1}, \ldots, \bar{C}_{4}$ be the $(-2)$-curves of $X^{\prime}$ such that $\bar{C}_{1}+\cdots+\bar{C}_{4}$ is even. Let $\bar{\Upsilon}$ be the proper transform of a general conic through $q_{1}$ and $q_{2}$ and suppose that $q_{i}, i=1,2$, is not infinitely near to $q_{0}$. Clearly $-2 K_{X^{\prime}} \equiv 2 \bar{\Upsilon}+\bar{C}_{1}+\cdots+\bar{C}_{4}$. Going back to $W$, setting $H$ and $\Upsilon$ the pull-back of $-K_{X^{\prime}}$ and $\bar{\Upsilon}$, respectively, then $C_{1}+\cdots+C_{4} \equiv 2(H-\Upsilon)$.

In Corollary 4.8 we saw that if $R$ has an irreducible component $\Gamma$ of genus 2, then $W$ is either an Enriques surface or a rational surface with $K_{W}^{2}=-2$ or -1 . Now we conclude this section by ruling out the case that $W$ is rational, as announced.

Proposition 7.10. Let $S$ be a numerical Godeaux surface with an involution (cf. notation in section (4)). Suppose that $-2 \leq K_{W}^{2} \leq-1$ and $\Gamma^{2}=1$. Then $W$ is not rational.

Proof. Suppose by contradiction that $W$ is rational. Let $\Gamma_{0}=\eta^{*}(\pi \Gamma)$. By the proof of Corollary 4.8, one has that $B_{0}-\Gamma_{0} \equiv-2 K_{W}$. Beauville's Lemma 3.11 and Theorem 4.1 imply that in this case $\sum_{i=1}^{4} C_{i}$ cannot be divisible by 2 .

By the above Lemma 7.1, it follows that $W^{\prime}=W$ and $K_{W}^{2}=-2$. In this case $B_{0}=\Gamma_{0}+N_{1}+N_{2}$, where $N_{1}, N_{2}$ are smooth rational curves with self-intersection -4 by formula (6). Then, because $B_{0}-\Gamma_{0} \equiv-2 K_{W}, N_{1}+N_{2} \equiv-2 K_{W}$. Note that $D-N_{1}-N_{2} \equiv D+2 K_{W}$ cannot be effective because $|M|=\left|D+K_{W}\right|$ is a base point free pencil of rational curves by Lemma 6.1

Since $\Gamma_{0} \in|D|$ is irreducible, the pencil $|D|$ has no fixed part. In particular any two distinct curves of $D$ have no common component. Since $D N_{1}=D N_{2}=0$, we can write $D \equiv G+N_{1} \equiv H+N_{2}$. Since, as we saw, $D-N_{1}-N_{2}$ is not effective, the two curves $G+N_{1}$ and $H+N_{2}$ in $|D|$ are distinct, hence $G, H$ are effective divisors without common components. Note that $G^{2}=H^{2}=-2$ and $K_{W} G=K_{W} H=-2$. Since $2 D \equiv G+H+N_{1}+N_{2} \equiv G+H-2 K_{W}$, one has $G+H \equiv 2\left(D+K_{W}\right) \equiv 2 M$.

By Lemma 6.1 every component $A$ of $G$ is either a (-1)-curve or a (-2)-curve. In the latter case, $0=A D=A H+A N_{2}$ implies that $A H=A N_{2}=0$, because $G$ has no common components with $H$. Since $N_{2} \equiv-2 K_{W}-N_{1}$, also $A N_{1}=0$, implying that $A G=0$. Moreover, since $K_{W} G=-2$, one has that $G$ contains either two (-1)-curves $A_{1}, A_{2}$ or one $(-1)$-curve $A$ with multiplicity 2 . In the first case, $A_{i} G \geq A_{i}^{2}=-1$, hence, since $G^{2}=-2$, the equality holds and $\left(G-A_{1}-A_{2}\right)^{2}=0$, implying by Zariski's Lemma that $G=A_{1}+A_{2}$. In the second case, one sees that $A G=-1$, implying that $A$ meets $G-2 A$ transversally at one point of a (-2)curve $\theta$. As above we conclude that $G=2 A+\theta$. Similarly one shows that either $H=A_{1}^{\prime}+A_{2}^{\prime}$ or $H=2 A^{\prime}+\theta^{\prime}$, where $A^{\prime}, A_{i}^{\prime}$ are $(-1)$-curves and $\theta^{\prime}$ is a (-2)-curve. 
Now recall that $2 M \equiv G+H, G$ and $H$ have no common components and $|D-M|=\emptyset$. So $G+H=M_{1}+M_{2}$, where $M_{1}, M_{2}$ are distinct fibres of $|M|$ and this excludes the possibility that $G=2 A+\theta$ or $H=2 A^{\prime}+\theta^{\prime}$.

Therefore the only remaining possibility is that $G=A_{1}+A_{2}$ and $H=A_{1}^{\prime}+A_{2}^{\prime}$. This implies that $M_{1}=A_{1}+A_{1}^{\prime}$ and $M_{2}=A_{2}+A_{2}^{\prime}$, after possibly reordering the indices, which is impossible by Corollary 6.2.

\section{On the torsion of numerical Godeaux surfaces of Du Val type}

In this section we will study the torsion of a numerical Godeaux surface $S$ of $\mathrm{Du}$ Val type. We will freely use the notation of the previous sections.

Proposition 8.1. Let $S$ be a numerical Godeaux surface of Du Val type. Then there is a non-trivial 2-torsion element in $\operatorname{Tors}(S)$, and accordingly, there is an étale 2-to-1 cover $\bar{S} \rightarrow S$, where $\bar{S}$ is a regular surface with $p_{g}(\bar{S})=1$ and $K_{\bar{S}}^{2}=2$. Therefore $\operatorname{Tors}(S)$ is either $\mathbb{Z} / 2 \mathbb{Z}$ or $\mathbb{Z} / 4 \mathbb{Z}$, depending on whether $\bar{S}$ has either no torsion or 2-torsion.

Proof. Recall that, by Remark 7.2 , the $(-2)$-curves $C_{1}, \ldots, C_{4}$ in $W$ are such that $C_{1}+\cdots+C_{4}$ is even and so $S$ has non-trivial 2-torsion, which defines an étale cover $\tilde{S} \rightarrow S$. The rest of the assertion follows from Theorem 4.1 .

We can now consider the following commutative diagram:

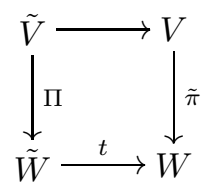

where $t: \tilde{W} \rightarrow W$ is the double cover branched over $C_{1}+\cdots+C_{4}, \Pi: \tilde{V} \rightarrow \tilde{W}$ is the double cover branched over $t^{*}\left(B_{0}+C_{5}\right)$ and $\tilde{V} \rightarrow V$ is the étale double cover associated to the 2-torsion of $V$.

Note that the minimal model of $\tilde{V}$ is obviously isomorphic to the surface $\tilde{S}$ of Proposition 8.1. Also remark that any smooth rational curve $\theta$ on $W$ disjoint from the curves $C_{1}, \ldots, C_{4}$ pulls back on $\tilde{W}$ to two disjoint rational curves with the same self-intersection number as $\theta$.

Note also that $t^{*}\left(B_{0}+C_{5}\right)=C_{5,1}+C_{5,2}+\tilde{B}_{0}$, where $\tilde{B}_{0}$ is an étale double cover of $B_{0}$, which is disjoint from the two $(-2)$-curves $C_{5,1}$ and $C_{5,2}$. Furthermore, since $B_{0}+C_{5}$ is divisible by 2 in $\operatorname{Pic}(W)$, then also $C_{5,1}+C_{5,2}+\tilde{B}_{0} \equiv 2 \Phi$ with $\Phi$ in $\operatorname{Pic}(\tilde{W})$.

Lemma 8.2. Let $S$ be a numerical Godeaux surface of Du Val type, and suppose that $\operatorname{Tors}(S)=\mathbb{Z} / 4 \mathbb{Z}$. Then the double cover $t$ splits over $B_{0}+C_{5}$.

Proof. By Proposition 8.1, $\tilde{V}$ has 2-torsion if and only if $\operatorname{Tors}(S)=\mathbb{Z} / 4 \mathbb{Z}$. On the other hand, $\tilde{W}$ is rational and thus, by Beauville's Lemma 3.11, $\tilde{V}$ has 2-torsion if and only if there exists a curve $G \supsetneqq \tilde{B}_{0}+C_{5,1}+C_{5,2}$ divisible by $2 \operatorname{in} \operatorname{Pic}(\tilde{W})$. Let $\iota$ be the involution on $\operatorname{Pic}(\tilde{W})$ (and $H^{2}(\tilde{W}, \mathbb{Z})$ ) induced by the involution on $\tilde{W}$ corresponding to the double cover $t$. We note that $\Phi$, where $C_{5,1}+C_{5,2}+\tilde{B}_{0} \equiv 2 \Phi$, is invariant under $\iota$.

Suppose that there exists a curve $G \supsetneqq \tilde{B}_{0}+C_{5,1}+C_{5,2}$ divisible by 2 in $\operatorname{Pic}(\tilde{W})$. Then also $H:=\tilde{B}_{0}+C_{5,1}+C_{5,2}-G$ is divisible by 2 , and we can write $G \equiv 2 L_{1}$, 
$H \equiv 2 L_{2}$, where $L_{1}+L_{2} \equiv \Phi$. Note that neither $L_{1}$ nor $L_{2}$ can be invariant under $\iota$, because otherwise there would be too many divisibility relations in the branch locus in $W$, implying again by Beauville's Lemma 3.11 that $\left|\operatorname{Tors}_{2}(S)\right| \geq 2$, which is impossible by Theorem 4.1 .

Then write $G=G^{\prime}+J_{1}$, and $H=H^{\prime}+J_{2}$, where all divisors appearing are effective, and we assume that $\iota\left(G^{\prime}\right)=G^{\prime}, \iota\left(H^{\prime}\right)=H^{\prime}, \iota\left(J_{i}\right) \neq J_{i}, i=1,2$ (and $\iota(\theta) \neq \theta$ for any component $\theta$ of $\left.J_{i}\right)$. Since $\iota(G+H)=G+H$, necessarily $\iota\left(J_{1}\right)=J_{2}$. Note also that $J_{i} \neq 0, i=1,2$, otherwise $\iota(G)=G$ and so $\iota\left(2 L_{1}\right)=2 \iota\left(L_{1}\right)=2 L_{1}$, implying, because $\tilde{W}$ is rational, that $\iota\left(L_{1}\right)=L_{1}$. This would mean that $\iota(G)=G$ and the divisibility would be coming already from $W$, which is impossible.

Now we have $G+\iota(G)=G^{\prime}+J_{1}+G^{\prime}+J_{2}=2 G^{\prime}+J_{1}+J_{2}$. On the other hand, $G+\iota(G) \equiv 2 L_{1}+2 \iota\left(L_{1}\right)$, and so we conclude that $J_{1}+J_{2} \equiv 2 L_{1}+2 \iota\left(L_{1}\right)-2 G^{\prime}$ is divisible by 2 . Again by Beauville's Lemma 3.11 and Theorem 4.1, there cannot further exist divisibility relations in $\tilde{B}_{0}+C_{5,1}+C_{5,2}$, hence we conclude that $G^{\prime}=$ $H^{\prime}=0, \iota\left(L_{1}\right)=L_{2}, \iota\left(J_{1}\right)=J_{2}$ and therefore $t$ splits over $B_{0}+C_{5}$.

Lemma 8.3. Let $S$ be a numerical Godeaux surface of Du Val type and suppose that the double cover $t$ splits over $f^{*}\left(B_{0}^{\prime}+C_{5}^{\prime}\right)$, where $f: W \rightarrow W^{\prime}$ is the birational morphism of Proposition 3.9, Then $\operatorname{Tors}(S)=\mathbb{Z} / 4 \mathbb{Z}$.

Proof. Note that the components of $f^{*}\left(B_{0}^{\prime}\right)$, which are not components of $B_{0}$, form trees of rational curves; each one of them is double in $f^{*}\left(B_{0}^{\prime}\right)$ and does not meet $C_{1}, \ldots, C_{4}$, so $t$ splits over it.

Write $t^{*}\left(f^{*}\left(B_{0}^{\prime}+C_{5}^{\prime}\right)\right)=A_{1}+C_{5,1}+A_{2}+C_{5,2}$ where $\iota\left(A_{1}\right)=A_{2}$ and $A_{i}, i=1,2$, is isomorphic to $f^{*}\left(B_{0}^{\prime}\right)$, so it is connected by Corollary 7.3, and each component of $A_{i}$, which is not a component of $t^{*}\left(B_{0}\right)$, is double in $A_{i}$. Recall that $B_{0}^{\prime}+C_{5}^{\prime} \equiv$ $4 \Delta-2 E+2 C_{5}^{\prime}$ by formula (15). Denote the two connected components of $t^{*}\left(f^{*}(E)\right)$ by $E_{1}$ and $E_{2}$, where $E_{1} C_{5,1}=1$ and $E_{1} C_{5,2}=0$. Since $E B_{0}^{\prime}=3$ and $\left(E+B_{0}^{\prime}\right)^{2}>0$, $t^{*}\left(f^{*}\left(E+B_{0}^{\prime}\right)\right)$ is connected and therefore, say, $E_{2} A_{1}=2$ and $E_{2} A_{2}=1$.

Then we claim that $A_{1} \equiv t^{*}\left(f^{*}(2 \Delta)\right)-2 E_{2}+C_{5,1}$. In fact we have $t^{*}(D) A_{1}=2$ and $t^{*}(D)\left(t^{*}\left(f^{*}(2 \Delta)\right)-2 E_{2}+C_{5,1}\right)=2$. Since $\left(A_{1}-t^{*}\left(f^{*}(2 \Delta)\right)+2 E_{2}-C_{5,1}\right)^{2}=0$, by the Index Theorem we obtain the claim, because $\tilde{W}$ is a rational surface.

Hence $A_{1}+C_{5,1}$ is divisible by 2 in $\operatorname{Pic}(\tilde{W})$, and therefore also $t^{*}\left(B_{0}+C_{5}\right)$ strictly contains an effective divisor divisible by 2 , implying by Beauville's Lemma 3.11 that $\tilde{V}$ (and $\tilde{S}$ ) has 2-torsion.

Remark 8.4. Note that if $t$ splits over $f^{*}\left(B_{0}^{\prime}+C_{5}^{\prime}\right)$, then $t$ splits also over $B_{0}+C_{5}$, but the converse is not necessarily true.

For the examples of the next section, we will need the following criterion.

Theorem 8.5. Let $S$ be a numerical Godeaux surface $S$ of Du Val type (cf., e.g., Corollary [7.6 and the notation therein). Suppose that $B_{0}$ on $W$ is a (smooth) irreducible curve with $p_{a}\left(B_{0}\right)=1$, and that $q_{i}, i=1,2$, is not infinitely near to $q_{0}$.

Then $\operatorname{Tors}(S)=\mathbb{Z} / 4 \mathbb{Z}$ if and only if there exists a (unique) plane curve of degree 8 with the following singularities:

- a point of multiplicity 2 at $q_{0}$;

- a point of type $[3,2]$ at $q_{i}, i=1,2$, where the infinitely near double point is in the direction of the line $r_{i}$;

- triple points at $q_{3}, q_{4}, q_{5}$;

- a tacnode at $q_{6}$, where the tacnodal tangent is also tangent to $B^{\bullet}$. 
Proof. The hypotheses imply that $W^{\prime}=W$. By Lemmas 8.2 8.3. Tors $(S)=\mathbb{Z} / 4 \mathbb{Z}$ if and only if the double cover $t$ splits on $B_{0}$, which happens by Remark 7.9 if and only if the restriction of $-\left(C_{1}+\cdots+C_{4}\right) / 2 \equiv-H+\Upsilon$ to $B_{0}$ is trivial, or, equivalently, if and only if $\mathcal{O}_{B_{0}}\left(K_{W}+B_{0}-H+\Upsilon\right) \cong \mathcal{O}_{B_{0}}$. Note that, since $B_{0} \cap C_{i}=\emptyset$, $i=1, \ldots, 4, \mathcal{O}_{B_{0}}\left(K_{W}+B_{0}-H+\Upsilon\right)$ has degree 0 . The sequence

$0 \rightarrow \mathcal{O}_{W}\left(K_{W}-H+\Upsilon\right) \rightarrow \mathcal{O}_{W}\left(K_{W}+B_{0}-H+\Upsilon\right) \rightarrow \mathcal{O}_{B_{0}}\left(K_{W}+B_{0}-H+\Upsilon\right) \rightarrow 0$ is exact. Note that $H^{0}\left(W, \mathcal{O}_{W}\left(K_{W}-H+\Upsilon\right)\right)=0$, because

$$
H^{0}\left(W, \mathcal{O}_{W}\left(2 K_{W}-C_{1}-\cdots-C_{4}\right)\right)=0,
$$

and, since $H^{2}\left(W, \mathcal{O}_{W}\left(K_{W}-H+\Upsilon\right)\right)=0$, the Riemann-Roch Theorem implies that $H^{1}\left(W, \mathcal{O}_{W}\left(K_{W}-H+\Upsilon\right)\right)=0$.

Thus $\mathcal{O}_{B_{0}}\left(K_{W}+B_{0}-H+\Upsilon\right) \cong \mathcal{O}_{B_{0}}$ if and only if $h^{0}\left(W, \mathcal{O}_{W}\left(K_{W}+B_{0}-H+\Upsilon\right)\right)=$ 1. The morphism $g^{\prime}: W \rightarrow \mathbb{P}^{2}$ (cf. the proof of Theorem [7.6) maps the unique curve in $\left|K_{W}+B_{0}-H+\Upsilon\right|$ to a plane curve of degree 8 as in the statement.

Theorem 8.5 can be extended, under suitable assumptions, e.g. as follows.

Corollary 8.6. Let $S$ be a numerical Godeaux surface $S$ of Du Val type. Suppose that $q_{i}, i=1,2$, is not infinitely near to $q_{0}$ and the curve $B_{0}$ on $W$ has two irreducible components, $\Gamma_{0}$ of genus 1 and $Z$ of genus 0 , such that $B_{0}^{\prime}$ on $W^{\prime}$ is the union of two curves meeting transversally at a point. Then $\operatorname{Tors}(S)=\mathbb{Z} / 4 \mathbb{Z}$ if and only if there exists a plane curve of degree 8, as in the statement of Theorem 8.5. containing the image of $Z$ in the plane.

Proof. Lemmas 8.2 and 8.3 again imply that the torsion group of $S$ is $\mathbb{Z} / 4 \mathbb{Z}$ if and only if the double cover $t: \tilde{W} \rightarrow W$ splits over $\Gamma_{0}$. The same argument of the proof of Theorem 8.5 shows that there is the plane curve of degree 8 as above and it contains the rational curve which is the image of $Z$ in the plane.

Remark 8.7. By Propositions 4.5 and 7.10 (cf. Remarks 6.11 and 7.7), it follows that numerical Godeaux surfaces described in Example 4.2 in $\mathrm{KL}$, which have Tors $=\mathbb{Z} / 4 \mathbb{Z}$, are birational to double planes of Du Val type. In that example, indeed, the ramification curve $R$ is irreducible of genus 1 , and this may happen, by our classification results, only if $W$ is rational and $S$ is of Du Val type.

A natural question is whether a numerical Godeaux surface $S$ of Du Val type can also be of Campedelli type, of course for different involutions on $S$.

In order to give a result about this problem, we first prove the following.

Proposition 8.8. Let $S$ be a numerical Godeaux surface of Campedelli type. If there is a non-trivial element $\eta_{2}$ in $\operatorname{Tors}_{2}(S)$, then the unique curve $\Delta_{2}$ in $\mid K_{S}+$ $\eta_{2} \mid$ is reducible and has a common component $A$ with the ramification curve $R$. Furthermore, $A$ is rational.

Proof. Note that $\Delta_{2}$ is fixed by the involution, and it is not contained in $R$, because $\Delta_{2}$ has arithmetic genus 2 (cf. Corollary 4.8 and Proposition 7.10). Suppose that $\Delta_{2}$ and $R$ have no common component. Looking at the Campedelli double plane representation (cf. Corollary 6.9), the image of $\Delta_{2}$ is a plane curve $\Delta^{\prime}$, which has no common component with the branch curve $B^{\bullet}$. Since $2 \Delta_{2} \in\left|2 K_{S}\right|$, the curve $2 \Delta^{\prime}$ is contained in the linear system of quartics with a double point at $p$ and passing simply through the five points of type $[3,3]$ with tangent lines also tangent to the [3,3]-point. Then $\Delta^{\prime}$ should be a conic passing through all the given 
points, a contradiction. We conclude that $\Delta_{2}$ and $R$ have a common component $A$. Reasoning as above, one sees that the image of $A$ in the plane is a conic, hence $A$ is rational.

Corollary 8.9. Let $S$ be a numerical Godeaux surface of Du Val type and $\pi^{\bullet}: V^{\bullet} \rightarrow$ $\mathbb{P}^{2}$ the corresponding double plane, branched along the curve $B^{\bullet}$ as in Corollary 7.6 . Let $\bar{\Delta}$ be the unique plane cubic curve passing through $q_{0}, q_{i}, i=1,2$, where it is tangent to $r_{i}, q_{3}, q_{4}, q_{5}$ and $q_{6}$. Suppose that $\bar{\Delta}$ is irreducible and does not pass through any irrelevant singularity of $B^{\bullet}$. Then $S$ has no involution which realizes it as a double plane of Campedelli type.

Proof. By Lemma 7.1 and Remark 7.2 $S$ has an element $\eta_{2}$ of 2-torsion. According to Remark 7.8, the unique curve $\Delta_{2}$ in $\left|K_{S}+\eta_{2}\right|$ is mapped to the plane cubic $\bar{\Delta}$. The hypotheses on $\bar{\Delta}$ imply that $\Delta_{2}$ is irreducible, hence there is no involution on $S$ which realizes it as a double plane of Campedelli type by Proposition 8.8 .

\section{Examples of numerical Godeaux surfaces of Du Val type}

As we already remarked, there was no previously known construction of numerical Godeaux surfaces as double planes of Du Val type.

In this section we produce such a construction. In order to do that, one has to find a reduced curve $B^{\bullet}$ of degree 12 with singularities at the points $q_{0}, \ldots, q_{6}$ as described in the Classification Theorem stated in the Introduction.

If one chooses the points in general position, then one expects no curve like $B^{\bullet}$, because the virtual dimension of the linear system of curve of degree 12 with those singularities is -2 . However it is possible to find such irreducible curves. One way is to look for a curve which is invariant under a linear transformation of the plane of order 2. This is an idea originally used by Stagnaro in order to construct numerical Godeaux surfaces as double planes of Campedelli type; cf. [St] and We3].

Example 9.1. Let $[x, y, z]$ be homogeneous coordinates in $\mathbb{P}^{2}$. Let $r_{1}$ be the line $x=y$ and choose the following points:

$$
q_{0}=[0,0,1], \quad q_{1}=[1,1,1], \quad q_{3}=[1,0,0], \quad q_{4}=[0,1,1], \quad q_{6}=[-2,0,1],
$$

and let $r_{3}$ be the line $x+2 z=0$, which passes through $q_{6}$. The linear involution

$$
\phi:[x, y, z] \in \mathbb{P}^{2} \rightarrow[x,-y, z] \in \mathbb{P}^{2}
$$

fixes $[0,1,0]$ and all the points of the line $y=0$. Consider the line $r_{2}=\phi\left(r_{1}\right)$ and the points $q_{2}=\phi\left(q_{1}\right)=[1,-1,1]$ and $q_{5}=\phi\left(q_{4}\right)=[0,-1,1]$.

Note that there is no conic passing through $q_{1}, q_{2}, \ldots, q_{6}$.

One sees that there are 49 monomials of degree 12 in $x, y, z$ whose equations are invariant under $\phi$, i.e. their degree in $y$ is even. Hence the curves of degree 12, which are invariant under $\phi$, form a linear system of dimension 48 . Now we impose:

- quadruple points at $q_{0}, q_{3}$ and $q_{4}$;

- a point of type $[4,4]$ at $q_{1}$, where the tangent direction is the line $r_{1}$;

- a point of type $[3,3]$ at $q_{6}$, where the tangent direction is the line $r_{3}$.

Note that, in this way, we are also imposing another quadruple point at $q_{5}=\phi\left(q_{4}\right)$ and another point of type $[4,4]$ at $q_{2}=\phi\left(q_{1}\right)$, where the tangent direction is the line $r_{2}=\phi\left(r_{1}\right)$, so the resulting curve should have the required singularities.

The interesting observation is that $q_{0}$ and $q_{3}$, instead of imposing 10 independent conditions each, impose only 6 conditions each, because of the symmetry of 
the configuration. For the same reason, the singularities at $q_{6}$ do not impose 12 independent conditions as expected, but only 6 . In conclusion, the number of independent conditions we are imposing is no more than 48 , so that there is surely a curve with at least the required singularities.

Using a computer algebra program (we used Maple for this), it turns out that there is one curve of degree 12 satisfying the above conditions and having exactly the required singularities, and not worse than those. Its equation is:

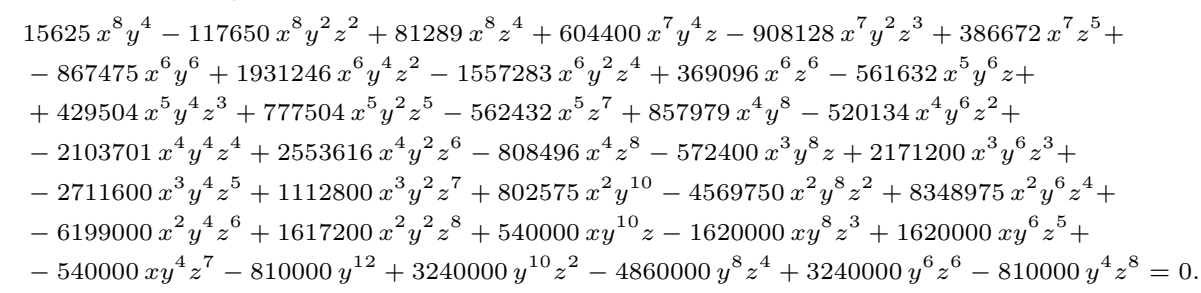

According to Maple, this curve is irreducible over the algebraic closure of $\mathbb{Q}$.

Proposition 9.2. The above curve of degree 12, together with the lines $x=y$ and $x=-y$, is the branch curve of a double plane whose smooth minimal model is a numerical Godeaux surface $S$ of Du Val type with $\operatorname{Tors}(S)=\mathbb{Z} / 4 \mathbb{Z}$.

Proof. By Theorem 8.5, it remains only to check that there exists a plane curve of degree 8 with the singularities described in that statement. Again using Maple, we found that such a curve actually exists and is the union of the sextic

$$
\begin{aligned}
& 900 y^{6}-300 y^{4} x z-1800 y^{4} z^{2}-519 y^{4} x^{2}+900 y^{2} z^{4}+106 y^{2} x^{3} z+1597 y^{2} x^{2} z^{2}+ \\
& -365 y^{2} x^{4}+300 y^{2} x z^{3}+130 x^{5} z-884 x^{2} z^{4}+299 x^{4} z^{2}-364 x^{3} z^{3}=0 .
\end{aligned}
$$

and the lines $y=z, y=-z$.

In We3, Caryn Werner shows that all numerical Godeaux surfaces of Campedelli type, whose branch curve is invariant under a linear involution of the plane, have $\operatorname{Tors}(S)=\mathbb{Z} / 4 \mathbb{Z}$. It would be interesting to see whether a similar statement is still true for numerical Godeaux surfaces of Du Val type.

The following example is computationally more complicated, but it also gives instances of numerical Godeaux surfaces $S$ of Du Val type with $\operatorname{Tors}(S)=\mathbb{Z} / 2 \mathbb{Z}$.

Example 9.3. Let $[x, y, z]$ be homogeneous coordinates in $\mathbb{P}^{2}$. Consider the lines $r_{1}: x=0, r_{2}: x=y$, and the points

$$
q_{0}=[0,0,1], q_{1}=[0,1,0], q_{2}=[1,1,0], q_{3}=[1,0,0], q_{4}=[-1,1,1], q_{5}=[1,-2,1] .
$$

We want to find a curve $B^{\bullet}$ which is reducible in the line $r_{3}: y=0$, which passes through $q_{0}$ and $q_{3}$, and in a curve $B_{1}^{\bullet}$ of degree 11 with the following singularities:

- two points of type $[4,4]$ in $q_{1}, q_{2}$, with tangent lines $r_{1}, r_{2}$;

- two quadruple points at $q_{4}$ and $q_{5}$;

- two triple points at $q_{0}, q_{3}$;

- a tacnode at a point $q_{6}=[t, 0,1]$ on $r_{3}$, with tacnodal tangent $r_{3}$.

Clearly, we want $q_{6}$ to be different from $q_{0}$, i.e. $t \neq 0$.

Note that the virtual dimension of the linear system of curves of degree 11 with these singularities is -1 . Thus it is reasonable to expect that for finitely many points on $r_{3}$ there is a curve with the required singularities. Using Maple, we found a polynomial $p(t)$ of degree 15 in $t$ such that, if $t_{0}$ is a root of $p(t)$, then there is a curve $B_{1}^{\bullet}$ with at least the above singularities, with $q_{6}=\left[t_{0}, 0,1\right] \neq q_{0}$. 
Luckily, the polynomial $p(t)$ factors over $\mathbb{Q}$, and there is an irreducible factor of degree 5 and another of degree 10. Maple is able to work out the computations on the corresponding algebraic extensions of $\mathbb{Q}$ and to verify that the corresponding curves $B_{1}^{\bullet}$ have the required, and not worse, singularities and are irreducible over the algebraic closure of $\mathbb{Q}$.

Unfortunately, as we said, the computations are complicated and the results are very cumbersome, and it is not the case to exhibit the explicit equations here. They can be found at the following Web address:

http://www.mat. uniroma2.it/ calabri/duValeqs.pdf.

It is interesting to remark that the solutions relative to the degree 5 factor have torsion $\mathbb{Z} / 4 \mathbb{Z}$, whereas the ones of the factor of degree 10 have torsion $\mathbb{Z} / 2 \mathbb{Z}$. This is verified by applying Corollary 8.6, i.e. by checking the existence or not of the curve of degree 8 with the appropriate singularities, which in this case is the union of the line $r_{3}$ and a curve of degree 7 .

Finally, one can check, again by using Maple, that the unique cubic plane curve $\bar{\Delta}$ as in Corollary 8.9 is irreducible and does not pass through the irrelevant singularities of $B^{\bullet}$, which is just a point in $B_{1}^{\bullet} \cap r_{3}$ different from $q_{1}, q_{3}, q_{6}$. Therefore these surfaces cannot be of Campedelli type by Corollary 8.9 .

Similar computations can also be tried in order to find irreducible curves $B^{\bullet}$, giving $\operatorname{Tors}(S)=\mathbb{Z} / 2 \mathbb{Z}$. Unfortunately, Maple is not able to carry out all the computations in a reasonable time. However we will see in a moment, in Corollary 9.7 that there are equisingular deformations of our curve which are irreducible.

Remark 9.4. Using Maple, we verified that, in both of the above examples, imposing all the required singularities but $q_{4}$, then the quadruple point $q_{4}$ can be chosen only in finitely many ways. The verification is performed by checking that there is no solution to the problem of finding the quadruple point $q_{4}$ on a given line, say $y-z=0$ in Example 9.1, and $x+y=0$ in Example 9.3 .

This agrees with the following fact. The linear system of curves of degree 12 with the given singularities at all the points but $q_{4}$ has virtual dimension 8 , which coincides with the actual dimension. This can be verified using Maple. Imposing a further fixed quadruple point $q_{4}$ is 10 more conditions. However, by moving $q_{4}$ with two parameters in the plane, we expect only eight more conditions. The above computation shows that this naive expectation is actually right.

We want to finish by giving information about the number of parameters on which our constructions of numerical Godeaux surfaces of Du Val type depend.

Proposition 9.5. Let $B^{\bullet}$ be the plane curve of degree 12 as in one of the two examples above. Let $\mathcal{V}$ be a complete family of plane curves of degree 12 which is maximal under the property that its general element is an equisingular deformation of $B^{\bullet}$. Then $\operatorname{dim} \mathcal{V}=13$.

Proof. It suffices to prove that $\operatorname{dim}(\mathcal{V} / P G L(3, \mathbb{C}))=5$. Up to projective transformations, we can fix distinct points $q_{0}, q_{1}, q_{2}$ and $q_{3}$, so that $r_{1}=\overline{q_{0} q_{1}}$ and $r_{2}=\overline{q_{0} q_{2}}$ are also fixed. Let $\mathcal{L}$ be the linear system of curves of degree 12 with quadruple points at $q_{0}, q_{3}$ and points of type $[4,4]$ at $q_{i}$, where the tangent line is $r_{i}, i=1,2$. Consider the following quadruplets $(B, p, q, \xi) \in \mathcal{L} \times \mathbb{P}^{2} \times \mathbb{P}^{2} \times \Delta$, where:

- $\Delta \subset \mathbb{P}^{2} \times \mathbb{P}^{2^{\star}}$ is the incidence correspondence so that $\xi \in \Delta$ is a pair $(x, r)$, with $x$ a point of the line $r$; 
- $B \in \mathcal{L}$ is irreducible and reduced;

- $q_{0}, \ldots, q_{3}, p, q, x$ are all distinct;

- $B$ has 4-uple points at $p, q$ and a [3,3]-point at $x$ with tangent direction $r$.

Let $\overline{\mathcal{V}}$ be an irreducible component of the closure of the set of such quadruplets, containing the point $\beta=\left(B^{\bullet}, q_{4}, q_{5},\left(q_{6}, r_{3}\right)\right)$. Note that $\operatorname{dim} \overline{\mathcal{V}} \geq 5$. In fact, $\bar{V}$ is defined in a neighbourhood of $\beta$ inside the variety $\mathcal{L} \times \mathbb{P}^{2} \times \mathbb{P}^{2} \times \Delta$, of dimension at least 37, by 32 equations. Since the projection on the first factor from $\overline{\mathcal{V}}$ to $\mathcal{L}$ is generically finite to its image, it suffices to prove that $\operatorname{dim} \overline{\mathcal{V}}=5$.

Then consider the projection $\rho: \overline{\mathcal{V}} \rightarrow \mathbb{P}^{2} \times \Delta$ to the last two factors. Remark 9.4 shows that $\rho$ is generically finite. This concludes the proof.

Corollary 9.6. The components of the moduli space of numerical Godeaux surfaces with an involution of Du Val type, containing our two Examples 9.1 and 9.3, are 5-dimensional.

Another interesting consequence is the following.

Corollary 9.7. In the case of Example 9.3 , the general element in $\overline{\mathcal{V}}$ corresponds to an irreducible curve.

Proof. Since, as we saw in the proof of Proposition 9.5, the map $\rho$ is generically finite, then it is also surjective. This means that the point $q_{6}$ can be moved out of the line passing through $q_{0}$ and $q_{3}$, hence, for the general member of $\overline{\mathcal{V}}$, the line cannot be a component of $B^{\bullet}$.

\section{REFERENCES}

[AS] M.F. Atiyah, I.M. Singer, The index of elliptic operators: III, Ann. of Math. 87 (1968), 546-604. MR0236952(38:5245)

[Bar1] R. Barlow, Some new surfaces with $p_{g}=$ 0, Duke Math. J. 51 (1984), no. 4, 889-904. MR 0771386 (86c:14032)

[Bar2] R. Barlow, A simply connected surface of general type with $p_{g}=0$, Invent. Math. 79 (1985), no. 2, 293-301. MR0778128 (87a:14033)

[Be] A. Beauville, Sur le nombre maximum de points doubles d'une surface dans $\mathbf{P}^{3}(\mu(5)=$ 31), in "Journées de Géométrie Algébrique d'Angers, Juillet 1979/Algebraic Geometry", Angers, 1979, pp. 207-215, Sijthoff \& Noordhoff, 1980. MR0605342 (82k:14037)

[BPV] W. Barth, C. Peters, A. Van de Ven, Compact complex surfaces, Ergebnisse der Mathematik und ihrer Grenzgebiete, 3. Folge, Band 4, Springer, 1984. MR.0749574 (86c:32026)

[Bl] S. Bloch, Lectures on algebraic cycles, Lecture one, Duke University Math. Series IV, Durham, 1980. MR.0558224 (82e:14012)

[Bo] G. Borrelli, On the classification of surfaces of general type with non-birational bicanonical map and Du Val double planes, preprint, math.AG n. 0312351.

$[\mathrm{CCM}]$ A. Calabri, C. Ciliberto, M. Mendes Lopes, Rational surfaces with an even set of four nodes, Math. Res. Lett. 11 (2004), n. 6, 799-808. MR2099361 (2006c:14061)

[CF] A. Calabri, R. Ferraro, Explicit resolutions of double points singularities of surfaces, Collect. Math. 53 (2002), n. 2, 99-131. MR1913513 (2003h:32043)

[Cam] L. Campedelli, Sopra alcuni piani doppi notevoli con curva di diramazione del decimo ordine, Atti Accad. Naz. Lincei 15 (1932), 536-542.

[CaD] F. Catanese, O. Debarre, Surfaces with $K^{2}=2, p_{g}=1, q=0$, J. Reine Angew. Math. 395 (1989), 1-55. MR0983058 (89m:14017)

[CL] F. Catanese, C. LeBrun, On the scalar curvature of Einstein manifolds, Math. Res. Lett. 4 (1997), no. 6, 843-854. MR1492124 (98k:53057)

[CP] F. Catanese, R. Pignatelli, On simply connected Godeaux surfaces, in T. Peternell, F.-O. Schreyer (eds.), Complex analysis and algebraic geometry. A volume in memory of Michael Schneider, 117-153, de Gruyter, 2000. MR.1760875 (2001g:14064) 
[Ci1] C. Ciliberto, The bicanonical map for surfaces of general type, in Algebraic geometrySanta Cruz 1995, 57-84, Proc. Sympos. Pure Math. 62, Part 1, Amer. Math. Soc. 1997. MR1492518 (98m:14040)

[Ci2] C. Ciliberto, The Geometry of Algebraic Varieties, in Development of Mathematics 19502000, edited by J.-P. Pier, Birkhäuser-Verlag, 2000. MR.1796844(2002k:14001)

[CoD] F. Cossec, I. Dolgachev, Enriques surfaces I, Progress in Math. 76, Birkhäuser, 1989. MR0986969 (90h:14052)

[CG] P.C. Craighero, R. Gattazzo, Quintic surfaces of $\mathbb{P}^{3}$ having a nonsingular model with $q=p_{g}=0, P_{2} \neq 0$, Rend. Sem. Mat. Univ. Padova 91 (1994), 187-198. MR1289636 (95g:14038)

[Do] I. Dolgachev, On algebraic surfaces with $q=p_{g}=0$, in G. Tomassini (ed.), Algebraic surfaces, III ciclo 1977, Villa Monastero-Varenna, Como. C.I.M.E., Liguori, 1988.

[DMP] I. Dolgachev, M. Mendes Lopes, R. Pardini, Rational surfaces with many nodes, Compositio Math. 132 (2002), no. 3, 349-363. MR1918136 (2003g:14049)

[DW] I. Dolgachev, C. Werner, A simply connected numerical Godeaux surface with ample canonical class, J. Algebraic Geom. 8 (1999), 737-764, with Erratum in J. Algebraic Geom. 10 (2001), 397. MR.1703612 (2000h:14030) MR.1811560 (2002a:14041)

[Du] P. Du Val, On surfaces whose canonical system is hyperelliptic, Canadian J. of Math. 4 (1952), 204-221. MR0048090 (13:977c)

[EV] H. Esnault, E. Viehweg, Lectures on vanishing theorems, DMV Seminar, Band 20, Birkhäuser, 1992. MR1193913 (94a:14017)

[Fr] M. Freedman, The topology of 4-manifolds, J. Differential Geometry 17 (1982), 357-454. MR0679066 (84b:57006)

[Go1] L. Godeaux, Sur une surface algébriques de genre zero et de bigenre deux, Atti Accad. Naz. Lincei 14 (1931), 479-481.

[Go2] L. Godeaux, Les surfaces algébriques non rationelles de genre arithmétique et géométriques nuls, Actulité Scientifiques et Industrielles 123, Exposés de Geométrie, IV, Hermann, Paris, 1934.

[Gr] M. Greenberg, Algebraic Topology: A First Course, W. A. Benjamin Publ., Reading, Mass., 1981. MR0643101 (83b:55001)

[Ho] E. Horikawa, On algebraic surfaces with pencils of curves of genus 2, in Complex analysis and algebraic geometry, 79-90, Iwanami Shoten, 1977. MR0453756 (56:12015)

[Ke] J.H. Keum, Some new surfaces of general type with $p_{g}=0$, preprint, 1988.

[KL] J.H. Keum, Y. Lee, Fixed locus of an involution acting on a Godeaux surface, Math. Proc. Cambridge Philos. Soc. 129 (2000), no. 2, 205-216. MR.1765910(2001f:14074)

[Ma] Maple 7, Waterloo Maple Inc., Waterloo, Ontario.

[Me] M. Mendes Lopes, Adjoint systems on surfaces, Boll. Un. Mat. Ital. A (7) 10 (1996), no. 1, 169-179. MR1386254 (97d:14011)

[MP1] M. Mendes Lopes, R. Pardini, The bicanonical map of surfaces with $p_{g}=0$ and $K^{2} \geq 7$, Bull. London Math. Soc. 33 (2001), 1-10. MR.1817764 (2002a:14042)

[MP2] M. Mendes Lopes, R. Pardini, A connected component of the moduli space of surfaces of general type with $p_{g}=0$, Topology 40 (2001), no. 5, 977-991. MR:1860538(2002g:14052)

[Mi] Y. Miyaoka, Tricanonical maps of numerical Godeaux surfaces, Invent. Math. 34 (1976), no. 2, 99-111. MR0409481 (53:13236)

[Mu M. Murakami, The torsion group of a certain numerical Godeaux surface, J. Math. Kyoto Univ. 41 (2001), no. 2, 323-333. MR1852987 (2002i:14040)

[Na] D. Naie, Surfaces d'Enriques et une construction de surfaces de type général avec $p_{g}=0$, Math. Z. 215 (2) (1994), 269-280. MR1259462 (94m:14055)

[Re1] M. Reid, Surfaces with $p_{g}=0, K^{2}=1$, J. Fac. Sci. Univ. Tokyo Sect. IA Math. 25 (1978), no. 1, 75-92. MR0494596 (80h:14018)

[Re2] M. Reid, Campedelli versus Godeaux, in Problems in the theory of surfaces and their classification (Cortona, 1988), 309-365, Sympos. Math. 32, Academic Press, 1991. MR1273384 (95h:14031)

[OP] F. Oort, C. Peters, A Campedelli surface with torsion group Z/2, Nederl. Akad. Wetensch. Indag. Math. 43 (1981), no. 4, 399-407. MR0639857(83h:14032)

[St] E. Stagnaro, On Campedelli branch loci, Ann. Univ. Ferrara Sez. VII (N.S.) 43 (1997), 1-26. MR1686746 (2000e:14061) 
[We1] C. Werner, A surface of general type with $p_{g}=q=0, K^{2}=1$, Manuscripta Math. 84 (1994), no. 3-4, 327-341. MR1291124 (95g:14042)

[We2] C. Werner, A four-dimensional deformation of a numerical Godeaux surface, Trans. Amer. Math. Soc. 349 (1997), no. 4, 1515-1525. MR1407503 (97k:14034)

[We3] C. Werner, Branch curves for Campedelli double planes, to appear in Rocky Mountain J. Math.

[Xi] G. Xiao, Surfaces fibrées en courbes de genre deux, Lecture Notes in Mathematics 1137, Springer, 1985. MR0872271 (88a:14042)

Dipartimento di Metodi e Modelli Matematici per le Scienze Applicate, Università degli Studi di Padova, via Trieste 63, I-35131 Padova, Italy

E-mail address: calabri@dmsa.unipd.it

Dipartimento di Matematica, Università degli Studi di Roma "Tor Vergata", Via Della Ricerca Scientifica, I-00133 Roma, Italy

E-mail address: cilibert@mat.uniroma2.it

Departamento de Matemática, Instituto Superior Técnico, Universidade Técnica de Lisboa, Av. Rovisco Pais, 1049-001 Lisboa, Portugal

E-mail address: mmlopes@math.ist.utl.pt 